Supporting Information

\title{
Corrole as a binucleating ligand: preparation, molecular structure and computational study of diboron corroles
}

\author{
Amelia M. Albrett, ${ }^{\mathrm{a}}$ Jeanet Conradie, ${ }^{\mathrm{b}, \mathrm{c}}$ Peter D. W. Boyd, ${ }^{\mathrm{a}}$ George \\ R. Clark, ${ }^{\mathrm{a}}$ Abhik Ghosh*, ${ }^{*}$ and Penelope J. Brothers, ${ }^{*, a}$
}

(a) Department of Chemistry, The University of Auckland, Private Bag 92019, Auckland, New Zealand; (b) Department of Chemistry and Center for Theoretical and Computational Chemistry, University of Troms $\phi, N-9037$ Troms $\phi$, Norway; (c) Department of Chemistry, University of the Free State, 9300 Bloemfontein, South Africa

\section{Contents}

Reproduction of Figure 2 with greater detail
A. Experimental procedures
B. Details of NMR spectra
C. UV-vis spectra
D. Details of Mass Spectra
E. Optimized Cartesian coordinates 
Figure 2. Relative energies (eV) and highlights of optimized (OLYP/TZ2P) bond lengths $(\AA$, in black), interatomic distances $(\AA$, in blue) and angles (deg, in red) of DFT-calculated structures of $\left[\mathrm{B}_{2} \mathrm{OF}_{2}(\mathrm{Cor})\right]^{-}$for (a) cisoid dipyrromethenes-only, (b) cisoid dipyrromethene/bipyrrole, (c) transoid dipyrromethenes-only, and (d) transoid dipyrromethene/bipyrrole coordination modes $(\AA, \mathrm{deg})$.

\begin{tabular}{|l|l|l|}
\hline Energy & Optimized geometry & Remark \\
\hline 0.0 & $\begin{array}{l}\text { (a) cisoid FBOBF unit } \\
\text { coordinated to } \\
\text { dipyrromethene binding sites }\end{array}$ \\
\hline 0.7 & $\begin{array}{l}\text { (b) cisoid FBOBF unit } \\
\text { coordinated to } \\
\text { dipyrromethene/dipyrrole } \\
\text { binding sites }\end{array}$ & (c) transoid FBOBF unit \\
coordinated to \\
dipyrromethene binding sites
\end{tabular}




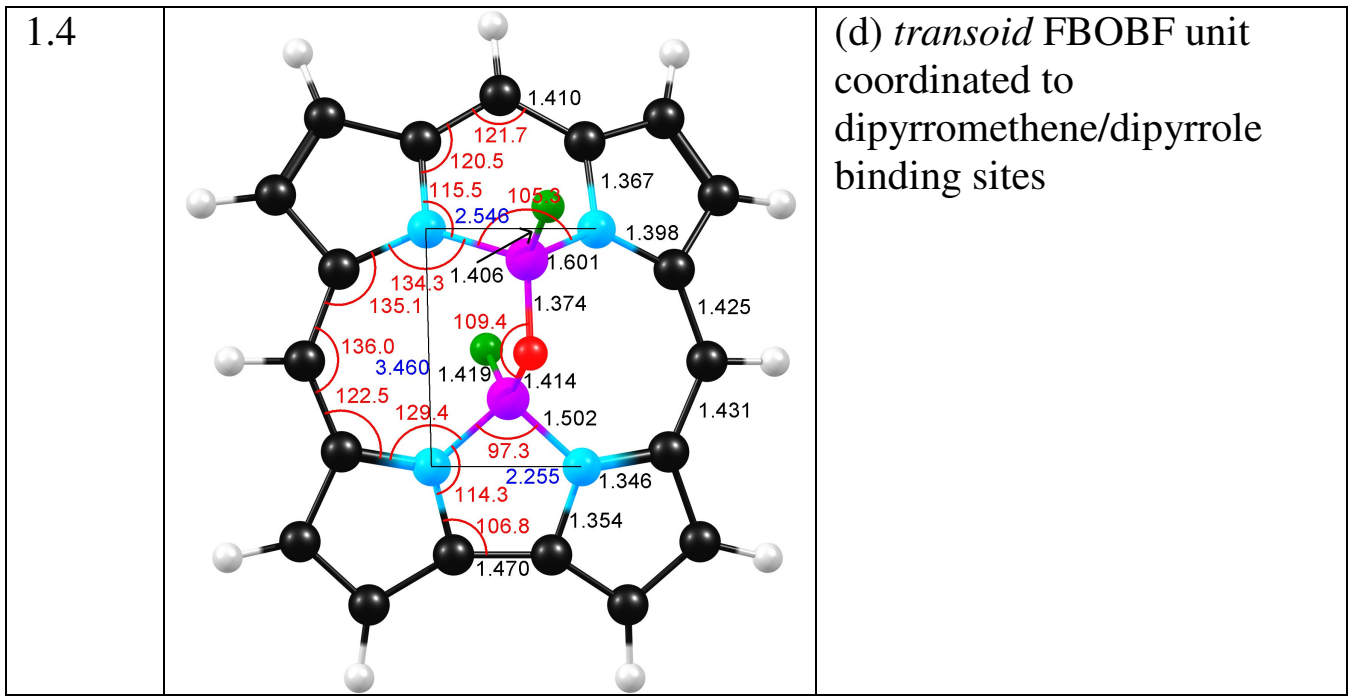




\section{A. Experimental procedure}

\section{Preparation of [HNEt $\left.\left({ }^{\mathrm{i}} \mathrm{Pr}_{2}\right)\right]\left[\left(\mathrm{B}_{2} \mathrm{OF}_{2}\right)(\mathrm{TPC})\right]$}

$\mathrm{BF}_{3} . \mathrm{Et}_{2} \mathrm{O}(0.177 \mathrm{~mL}, 1.44 \mathrm{mmol})$ was added dropwise via syringe to a solution of TPC (63.0 mg, $1.2 \mathrm{mmol})$ in dichloromethane $(15 \mathrm{~mL})$, under nitrogen.

Diisopropylethylamine $(0.417 \mathrm{~mL}, 2.39 \mathrm{mmol})$ was added dropwise and the green/ purple dichroic solution stirred at room temperature for $12 \mathrm{~h}$. n-Hexane $(5 \mathrm{~mL})$ was added and the resulting white precipitate removed by filtration. The filtrate was collected and recrystallised several times from dichloromethane/n-hexane to remove all white precipitate. The filtrate was collected then the solvent removed in vacuo. The purple solid was dissolved in dichloromethane $(5 \mathrm{~mL})$ and $\mathrm{n}$-hexane $(2 \mathrm{~mL})$ and left to crystallise under nitrogen over 1 week. The mother liquor was removed by pipette then the crystals were washed several times with n-hexane, then dried in vacuo.

Yield $35 \mathrm{mg}, 40 \%$. Anal. Found: C 73.65, H 6.01, N 9.71\%. Calcd for $\mathrm{C}_{45} \mathrm{H}_{43} \mathrm{~B}_{2} \mathrm{~F}_{2} \mathrm{~N}_{5} \mathrm{O} 0.2\left(\mathrm{H}_{2} \mathrm{O}\right): \mathrm{C} 73.73, \mathrm{H} 5.97, \mathrm{~N} 9.55 \%$. MS (electrospray ionization, anion, major isotopomer): Found 599.203, Calcd 599.203. ${ }^{1} \mathrm{H} \mathrm{NMR}\left(\mathrm{CDCl}_{3}, 400\right.$ $\mathrm{MHz}): \delta(\mathrm{ppm})=0.01-0.03\left(6 \mathrm{H}, \mathrm{d}, \mathrm{J}=6.40 \mathrm{~Hz}, \mathrm{NCH}\left(\mathrm{CH}_{3}\right)_{2}\right), 0.06(1 \mathrm{H}, \mathrm{s}, \mathrm{NH})$, $0.16-0.20\left(3 \mathrm{H}, \mathrm{t}, \mathrm{J}=14.4 \mathrm{~Hz}, \mathrm{NCH}_{2} \mathrm{CH}_{3}\right), 0.25-0.27(6 \mathrm{H}, \mathrm{d}, \mathrm{J}=6.40 \mathrm{~Hz}$, $\left.\mathrm{NCH}\left(\mathrm{CH}_{3}\right)_{2}\right), 1.72\left(2 \mathrm{H}, \mathrm{m}, \mathrm{NCH}_{2} \mathrm{CH}_{3}\right), 2.20\left(2 \mathrm{H}, \mathrm{m}, \mathrm{NCH}\left(\mathrm{CH}_{3}\right)_{2}\right), 7.51-7.55(2 \mathrm{H}, \mathrm{t}$, $\mathrm{J}=7.44 \mathrm{~Hz}, 5,15$-para $), 7.61-7.65(4 \mathrm{H}, \mathrm{t}, \mathrm{J}=7.32 \mathrm{~Hz}, 5,15-$ meta $), 7.64-7.69(2 \mathrm{H}$, $\mathrm{t}, \mathrm{J}=7.28 \mathrm{~Hz}, 10-$ meta $), 7.70-7.74(1 \mathrm{H}, \mathrm{t}, \mathrm{J}=6.12 \mathrm{~Hz}, 10$-para $), 8.01-8.03(2 \mathrm{H}, \mathrm{d}$, $\mathrm{J}=7.88 \mathrm{~Hz}, 10$-ortho $), 8.09-8.11(4 \mathrm{H}, \mathrm{d}, \mathrm{J}=8.32 \mathrm{~Hz}, 5,15$-ortho $), 8.11-8.12(2 \mathrm{H}$, $\mathrm{d}, \mathrm{J}=4.36 \mathrm{~Hz}, 3,17-\beta), 8.34-8.35(2 \mathrm{H}, \mathrm{d}, \mathrm{J}=5.76 \mathrm{~Hz}, 7,13-\beta), 8.47-8.48(2 \mathrm{H}, \mathrm{d}, \mathrm{J}$ $=4.52 \mathrm{~Hz}, 8,12-\beta), 8.94-8.96(2 \mathrm{H}, \mathrm{d}, \mathrm{J}=4.32 \mathrm{~Hz}, 2,18-\beta) .{ }^{19} \mathrm{~F} \mathrm{NMR}\left(\mathrm{CDCl}_{3}, 376\right.$ $\mathrm{MHz}): \delta(\mathrm{ppm})=-131.70(\mathrm{br} \mathrm{s}) .{ }^{11} \mathrm{~B} \mathrm{NMR}\left(\mathrm{CDCl}_{3}, 128 \mathrm{MHz}\right): \delta(\mathrm{ppm})=-10.08(\mathrm{br}$ s). UV-vis $\left(\lambda_{\max } / \mathrm{nm}\left(\varepsilon / \mathrm{M}^{-1} \mathrm{~cm}^{-1}\right)\right.$ in $\left.\mathrm{CH}_{2} \mathrm{Cl}_{2}\right)$ : 418 (89871), 421 (89796), 489 (6669), 531 (4959), 572 (6849), 603 (11379).

\section{Preparation of $\left[\mathrm{HNEt}\left({ }^{\mathrm{i}} \mathrm{Pr}_{2}\right)\right]\left[\left(\mathrm{B}_{2} \mathrm{OF}_{2}\right)\{\mathrm{T}(4-\mathrm{F}-\mathrm{P}) \mathrm{C}\}\right]$}

Synthetic procedure same as for $\left[\mathrm{HNEt}\left({ }^{\mathrm{i}} \mathrm{Pr}_{2}\right)\right]\left[\left(\mathrm{B}_{2} \mathrm{OF}_{2}\right)(\mathrm{TPC})\right]$.

Yield $32 \%$. Anal. Found: C, 69.89; H, 5.41; N, 9.11\%. Calcd for $\mathrm{C}_{45} \mathrm{H}_{40} \mathrm{~B}_{2} \mathrm{~F}_{5} \mathrm{~N}_{5} \mathrm{O} 0.3\left(\mathrm{NEt}\left({ }^{\mathrm{i}} \mathrm{Pr}\right)_{2}\right): \mathrm{C}, 69.36 ; \mathrm{H}, 5.64 ; \mathrm{N}, 9.02 \%$. MS (electrospray ionization, anion, major isotopomer): Found 653.175, Calcd 653.174. ${ }^{1} \mathrm{H}$ NMR $\left(\mathrm{CDCl}_{3}, 400 \mathrm{MHz}\right): \delta(\mathrm{ppm})=1.33-1.42\left(15 \mathrm{H}, \mathrm{m}, \mathrm{NCH}\left(\mathrm{CH}_{3}\right)_{2}, \mathrm{NCH}_{2} \mathrm{CH}_{3}\right), 1.50-$ $1.70(1 \mathrm{H}$, br s, $\mathrm{NH}), 2.99-3.05\left(2 \mathrm{H}, \mathrm{q}, \mathrm{NCH}_{2} \mathrm{CH}_{3}\right), 3.54-3.61(2 \mathrm{H}, \mathrm{m}$, $\left.\mathrm{NCH}\left(\mathrm{CH}_{3}\right)_{2}\right), 7.31$ - 7.36 (4H, m, 5,15-meta), 7.35 - 7.39 (2H, m, 10-meta), 7.93 $7.95(2 \mathrm{H}, \mathrm{m}, 10$-ortho $), 8.01-8.04(4 \mathrm{H}, \mathrm{m}, 5,15$-ortho $), 8.06-8.07(2 \mathrm{H}, \mathrm{d}, \mathrm{J}=4.44$ $\mathrm{Hz}, 3,17-\beta), 8.29-8.30(2 \mathrm{H}, \mathrm{d}, \mathrm{J}=4.44 \mathrm{~Hz}, 13,17-\beta), 8.44-8.46(2 \mathrm{H}, \mathrm{d}, \mathrm{J}=4.44$ $\mathrm{Hz}, 8,12-\beta), 8.95-8.96(2 \mathrm{H}, \mathrm{d}, \mathrm{J}=4.40 \mathrm{~Hz}, 2,18-\beta) .{ }^{19} \mathrm{~F} \mathrm{NMR}\left(\mathrm{CDCl}_{3}, 376 \mathrm{MHz}\right): \delta$ $(\mathrm{ppm})=-132.00(\mathrm{br} \mathrm{s}, \mathrm{BF}),-117.1\left(\mathrm{~s}, \mathrm{C}_{6} \mathrm{H}_{4} \mathrm{~F}\right),-117.6\left(\mathrm{~s}, 2 \mathrm{xC}_{6} \mathrm{H}_{4} \mathrm{~F}\right) .{ }^{11} \mathrm{~B}$ NMR $\left(\mathrm{CDCl}_{3}, 128 \mathrm{MHz}\right): \delta(\mathrm{ppm})=-10.06(\mathrm{br} \mathrm{s})$. UV-vis $\left(\lambda_{\max } / \mathrm{nm}\left(\varepsilon / \mathrm{M}^{-1} \mathrm{~cm}^{-1}\right)\right.$ in $\mathrm{CH}_{2} \mathrm{Cl}_{2}$ ): 416 (98066), 530 (6575), 570 (9296), 601 (12698).

\section{Preparation of $\left[\mathrm{HNEt}\left({ }^{\mathrm{i}} \operatorname{Pr}_{2}\right)\right]\left[\left(\mathrm{B}_{2} \mathrm{OF}_{2}\right)\left\{\mathbf{T}\left(4-\mathrm{CF}_{3}-\mathrm{P}\right) \mathrm{C}\right\}\right]$}

Synthetic procedure same as for $\left[\mathrm{HNEt}\left({ }^{\mathrm{i}} \mathrm{Pr}_{2}\right)\right]\left[\left(\mathrm{B}_{2} \mathrm{OF}_{2}\right)(\mathrm{TPC})\right]$ 
Yield $34 \%$. Anal. Found: C, 60.33; H, 4.42; N, $7.34 \%$. Calcd for $\mathrm{C}_{48} \mathrm{H}_{40} \mathrm{~B}_{2} \mathrm{~F}_{11} \mathrm{~N}_{5} \mathrm{O} \cdot \mathrm{H}_{2} \mathrm{O}: \mathrm{C}, 60.59 ; \mathrm{H}, 4.45 ; \mathrm{N}, 7.36 \%$. MS (electrospray ionization, anion, major isotopomer): Found 803.165, Calcd 803.165. ${ }^{1} \mathrm{H} \mathrm{NMR}\left(\mathrm{CDCl}_{3}, 400\right.$ $\mathrm{MHz}): \delta(\mathrm{ppm})=0.30-0.35(1 \mathrm{H}$, br s, $\mathrm{NH}), 0.91-0.97\left(15 \mathrm{H}, \mathrm{m}, \mathrm{NCH}\left(\mathrm{CH}_{3}\right)_{2}\right.$, $\left.\mathrm{NCH}_{2} \mathrm{CH}_{3}\right), 2.55\left(2 \mathrm{H}, \mathrm{m}, \mathrm{NCH}_{2} \mathrm{CH}_{3}\right), 3.06\left(2 \mathrm{H}, \mathrm{m}, \mathrm{NCH}\left(\mathrm{CH}_{3}\right)_{2}\right), 7.90-7.92(4 \mathrm{H}, \mathrm{d}, \mathrm{J}$ $=8.28 \mathrm{~Hz}, 5,15-$ meta $), 7.96-7.98(2 \mathrm{H}, \mathrm{d}, \mathrm{J}=8.00 \mathrm{~Hz}, 10-$ meta $), 8.10-8.12(2 \mathrm{H}, \mathrm{d}, \mathrm{J}$ $=4.40 \mathrm{~Hz}, 3,17-\beta), 8.13-8.15(2 \mathrm{H}, \mathrm{J}=8.00 \mathrm{~Hz}, 10$-ortho $), 8.20-8.22(4 \mathrm{H}, \mathrm{d}, \mathrm{J}=$ $7.20 \mathrm{~Hz}, 5,15$-ortho $), 8.32-8.33(2 \mathrm{H}, \mathrm{d}, \mathrm{J}=4.52 \mathrm{~Hz}, 7,13-\beta), 8.43-8.45(2 \mathrm{H}, \mathrm{d}, \mathrm{J}=$ $4.52 \mathrm{~Hz}, 8,12-\beta), 9.00-9.01(2 \mathrm{H}, \mathrm{d}, \mathrm{J}=4.44 \mathrm{~Hz}, 2,18-\beta) .{ }^{19} \mathrm{~F} \mathrm{NMR}\left(\mathrm{CDCl}_{3}, 376\right.$ $\mathrm{MHz}): \delta(\mathrm{ppm})=-131.98(\mathrm{br} \mathrm{s}, \mathrm{BF}),-63.07\left(\mathrm{~s}, \mathrm{CF}_{3}\right),-63.16\left(\mathrm{~s}, 2 \mathrm{xCF}_{3}\right) .{ }^{11} \mathrm{~B} \mathrm{NMR}$ $\left(\mathrm{CDCl}_{3}, 128 \mathrm{MHz}\right): \delta(\mathrm{ppm})=-10.09$ (br s). UV-vis $\left(\lambda_{\max } / \mathrm{nm}\left(\varepsilon / \mathrm{M}^{-1} \mathrm{~cm}^{-1}\right)\right.$ in $\mathrm{CH}_{2} \mathrm{Cl}_{2}$ ): 421 (103597), 534 (5232), 572 (7895), 601 (11549).

\section{Preparation of [HNEt $\left.\left({ }^{i} \operatorname{Pr}_{2}\right)\right]\left[\left(\mathrm{B}_{2} \mathrm{OF}_{2}\right)\left\{\mathbf{T}\left(4-\mathrm{CH}_{3}-\mathrm{P}\right) \mathrm{C}\right\}\right]$}

Synthetic procedure same as for $\left[\mathrm{HNEt}\left({ }^{\mathrm{i}} \mathrm{Pr}_{2}\right)\right]\left[\left(\mathrm{B}_{2} \mathrm{OF}_{2}\right)(\mathrm{TPC})\right]$

Yield $35 \%$. Anal. Found: C 67.00, H 6.15, N 7.86\%. Calcd for $\mathrm{C}_{48} \mathrm{H}_{49} \mathrm{~B}_{2} \mathrm{~F}_{2} \mathrm{~N}_{5} \mathrm{O} 4.7\left(\mathrm{H}_{2} \mathrm{O}\right): \mathrm{C} 67.33, \mathrm{H} 6.87$, N 8.18\%MS (electrospray ionization, anion, major isotopomer): Found 641.251, Calcd 641.250. ${ }^{1} \mathrm{H} \mathrm{NMR}\left(\mathrm{CDCl}_{3}, 300\right.$ $\mathrm{MHz}): \delta(\mathrm{ppm})=0.058(1 \mathrm{H}, \mathrm{br} \mathrm{s}, \mathrm{NH}), 0.85-0.95\left(15 \mathrm{H}, \mathrm{m}, \mathrm{NCH}\left(\mathrm{CH}_{3}\right)_{2}\right.$, $\left.\mathrm{NCH}_{2} \mathrm{CH}_{3}\right), 2.42-2.50\left(2 \mathrm{H}, \mathrm{m}, \mathrm{NCH}_{2} \mathrm{CH}_{3}\right), 2.55\left(6 \mathrm{H}, \mathrm{s}, \mathrm{C}_{6} \mathrm{H}_{6} \mathrm{CH}_{3}\right), 2.68(3 \mathrm{H}, \mathrm{s}$, $\left.\mathrm{C}_{6} \mathrm{H}_{6} \mathrm{CH}_{3}\right), 3.00-3.10\left(2 \mathrm{H}, \mathrm{m}, \mathrm{NCH}\left(\mathrm{CH}_{3}\right)_{2}\right), 7.42-7.46(4 \mathrm{H}, \mathrm{d}, \mathrm{J}=8.6 \mathrm{~Hz}, 5,15-$ meta $), 7.46-7.49(2 \mathrm{H}, \mathrm{d}, \mathrm{J}=7.94 \mathrm{~Hz}, 10-m e t a), 7.88-7.91(2 \mathrm{H}, \mathrm{d}, \mathrm{J}=8.03 \mathrm{~Hz}, 10$ ortho $), 7.97-8.00(4 \mathrm{H}, \mathrm{d}, \mathrm{J}=7.90 \mathrm{~Hz}, 5,15$-ortho $), 8.08-8.10(2 \mathrm{H}, \mathrm{d}, \mathrm{J}=4.27 \mathrm{~Hz}$, $3,17-\beta), 8.33-8.35(2 \mathrm{H}, \mathrm{d}, \mathrm{J}=4.28 \mathrm{~Hz}, 7,13-\beta), 8.48-8.49(2 \mathrm{H}, \mathrm{d}, \mathrm{J}=4.52 \mathrm{~Hz}$, $8,12-\beta), 8.92-8.93(2 \mathrm{H}, \mathrm{d}, \mathrm{J}=4.34 \mathrm{~Hz}, 2,18-\beta) .{ }^{19} \mathrm{~F} \mathrm{NMR}\left(\mathrm{CDCl}_{3}, 376 \mathrm{MHz}\right): \delta$ $(\mathrm{ppm})=-131.97$ (br s). ${ }^{11} \mathrm{~B} \mathrm{NMR}\left(\mathrm{CDCl}_{3}, 128 \mathrm{MHz}\right): \delta(\mathrm{ppm})=-10.02$ (br s). UVvis $\left(\lambda_{\max } / \mathrm{nm}\left(\varepsilon / \mathrm{M}^{-1} \mathrm{~cm}^{-1}\right)\right.$ in $\left.\mathrm{CH}_{2} \mathrm{Cl}_{2}\right): 415$ (112376), 530 (8436), 565 (10456), 605 (15681). 
B. Details of NMR spectra

B1. NMR spectra of $\left.\left[\mathrm{HNEt}^{(\mathrm{i}} \mathrm{Pr}_{2}\right)\right]\left[\left(\mathrm{B}_{2} \mathrm{OF}_{2}\right) \mathrm{TPC}\right]$

$\underline{\text { Labeling scheme for assignments }}$
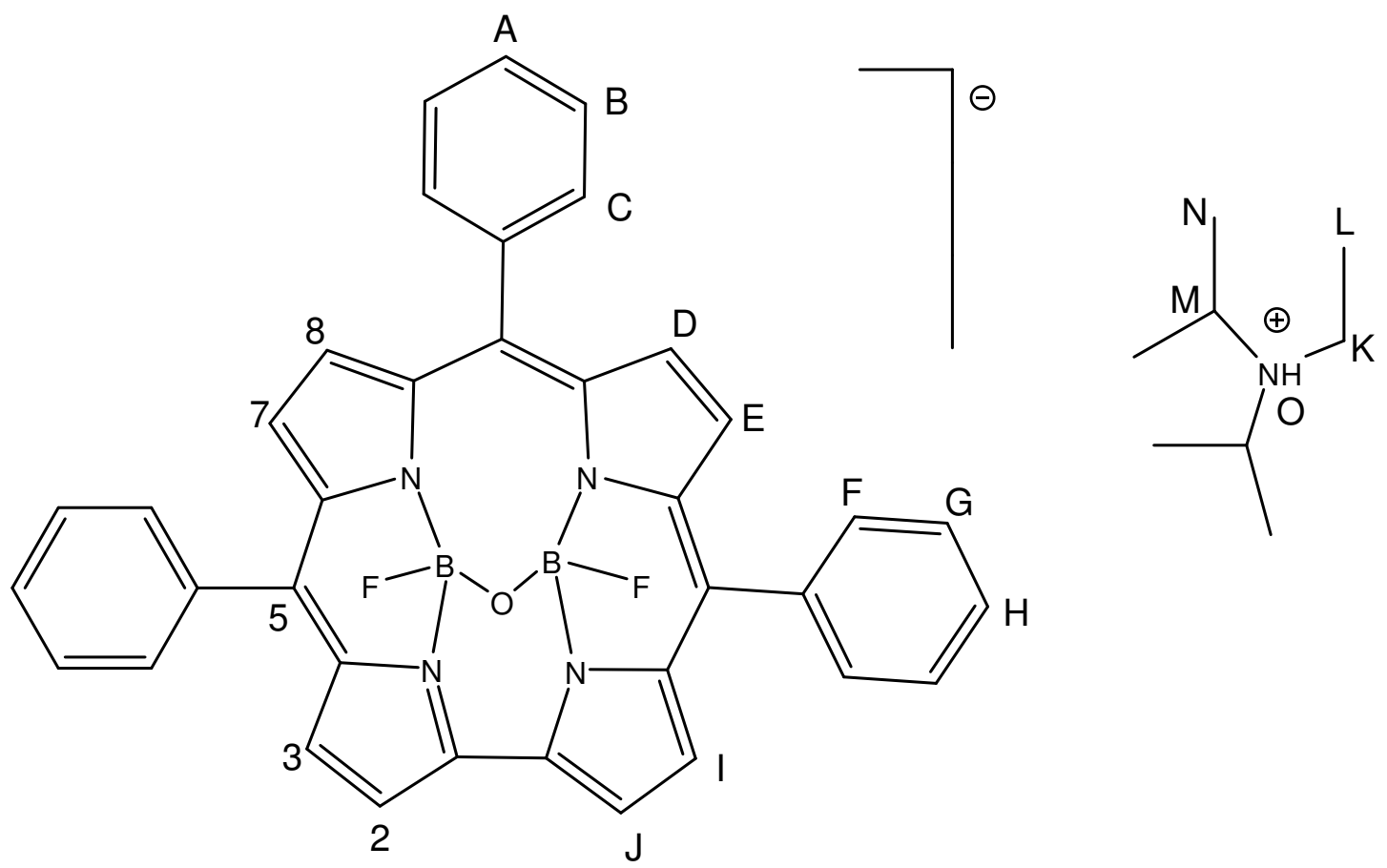
.. $\left[\mathrm{HNEt}\left({ }^{\mathrm{i}} \operatorname{Pr}_{2}\right)\right]\left[\left(\mathrm{B}_{2} \mathrm{OF}_{2}\right) \mathrm{TPC}\right]$ continued

1H NMR:

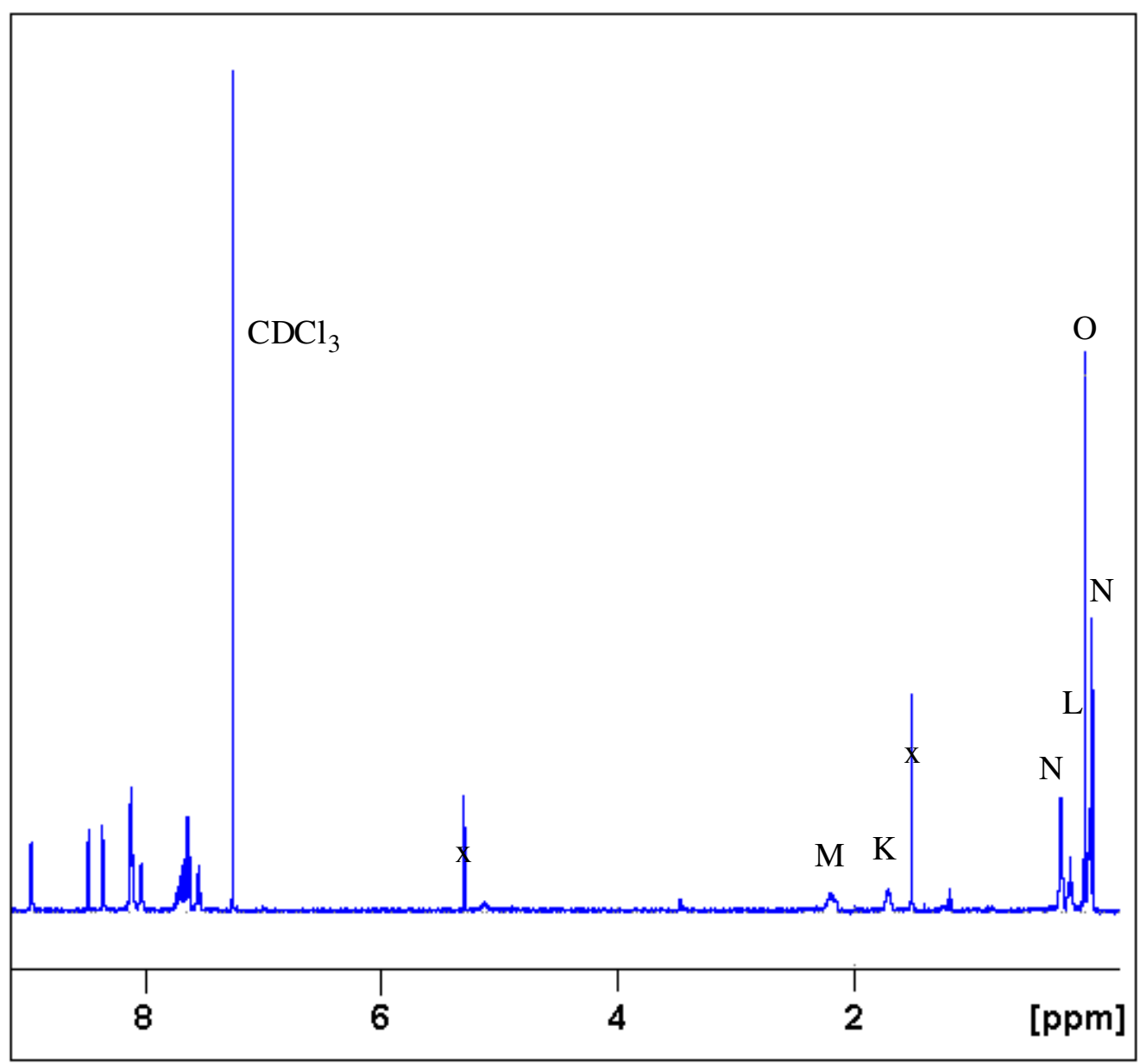


.. $\left[\mathrm{HNEt}\left({ }^{\mathrm{i}} \operatorname{Pr}_{2}\right)\right]\left[\left(\mathrm{B}_{2} \mathrm{OF}_{2}\right) \mathrm{TPC}\right]$ continued

${ }^{1}$ H NMR (aromatic region):

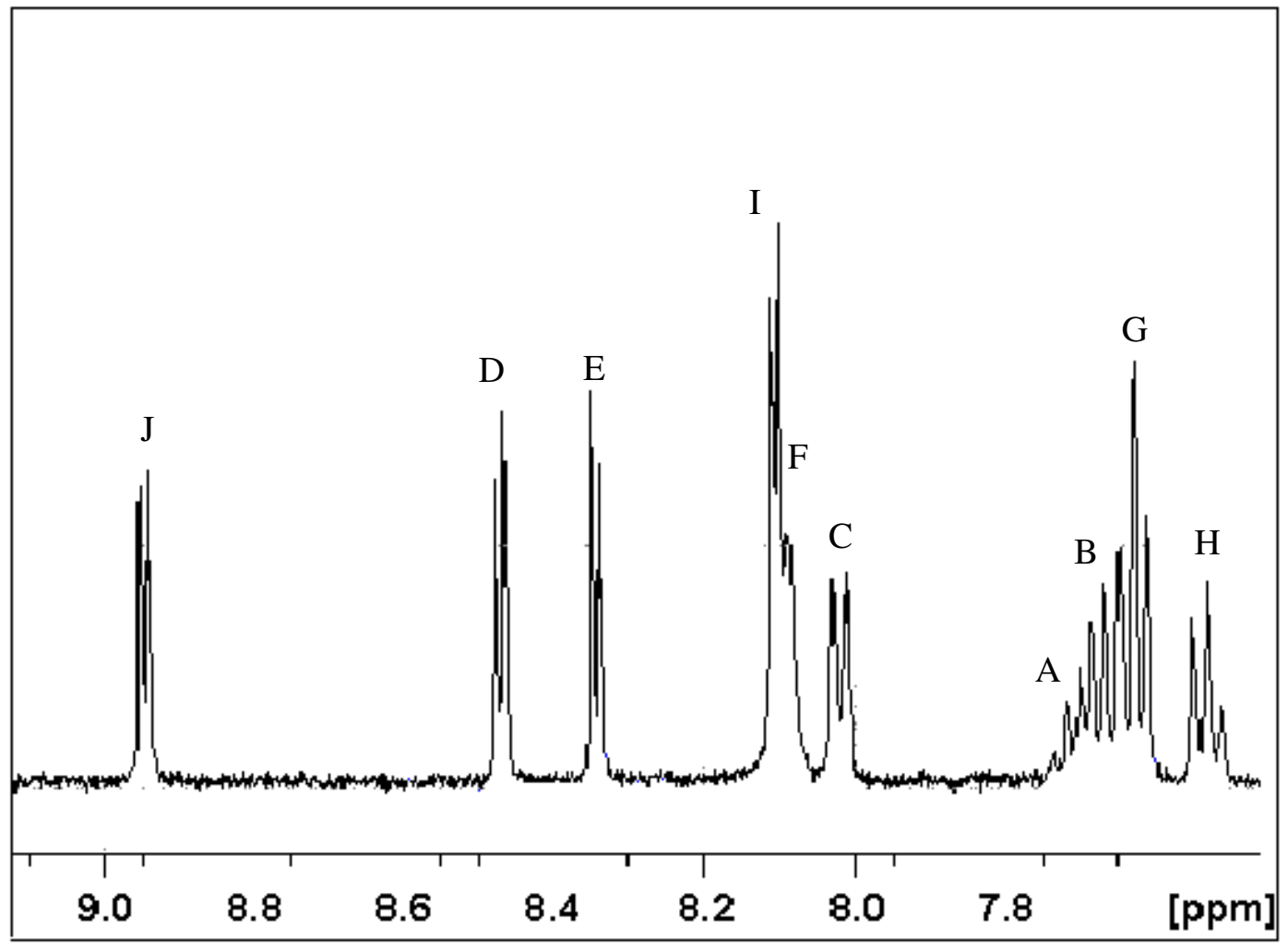


.. $\left[\mathrm{HNEt}\left({ }^{\mathrm{i}} \operatorname{Pr}_{2}\right)\right]\left[\left(\mathrm{B}_{2} \mathrm{OF}_{2}\right) \mathrm{TPC}\right]$ continued

\section{COSY:}

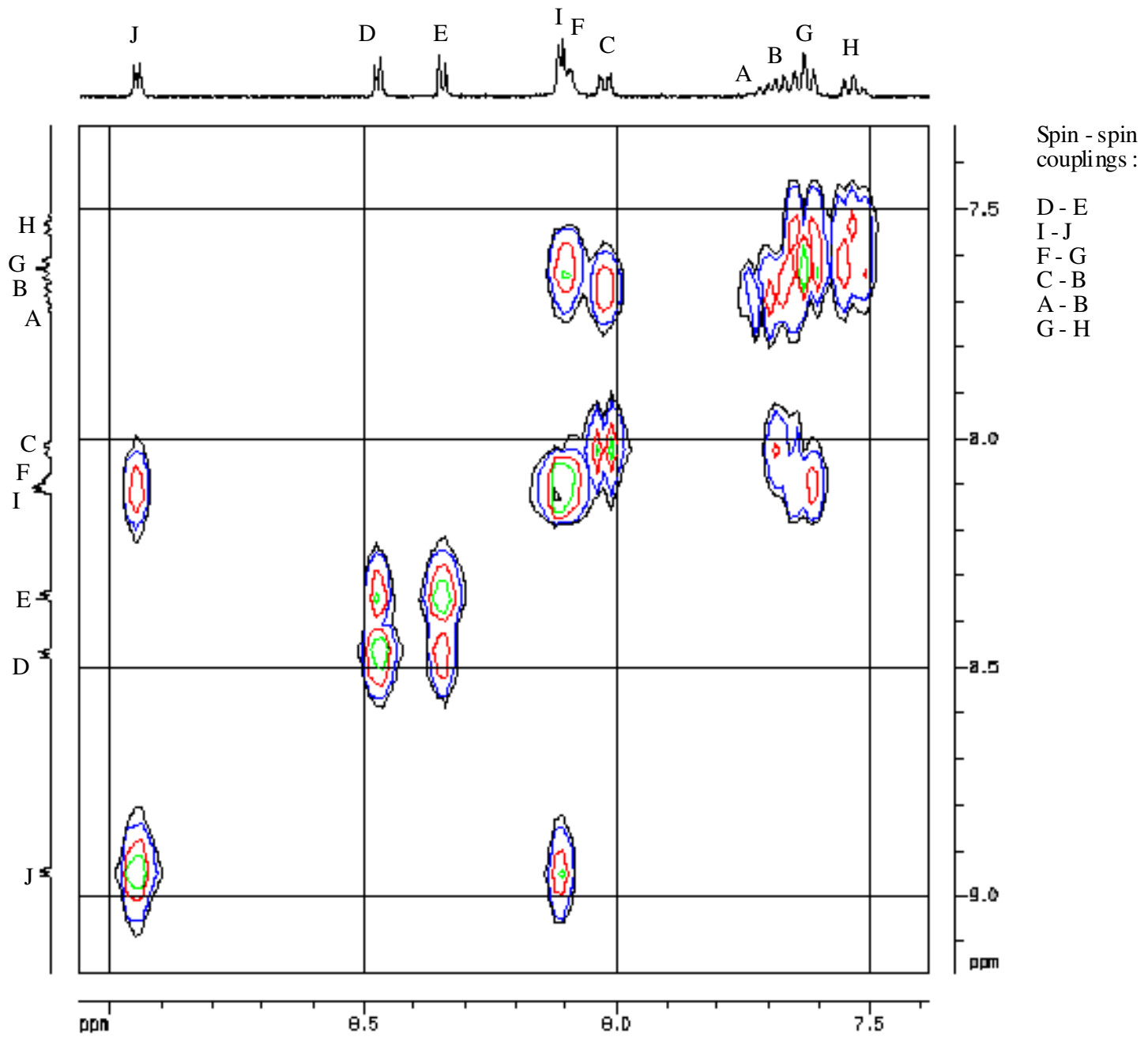


.. $\left[\mathrm{HNEt}\left({ }^{\mathrm{i}} \operatorname{Pr}_{2}\right)\right]\left[\left(\mathrm{B}_{2} \mathrm{OF}_{2}\right) \mathrm{TPC}\right]$ continued

\section{NOESY:}

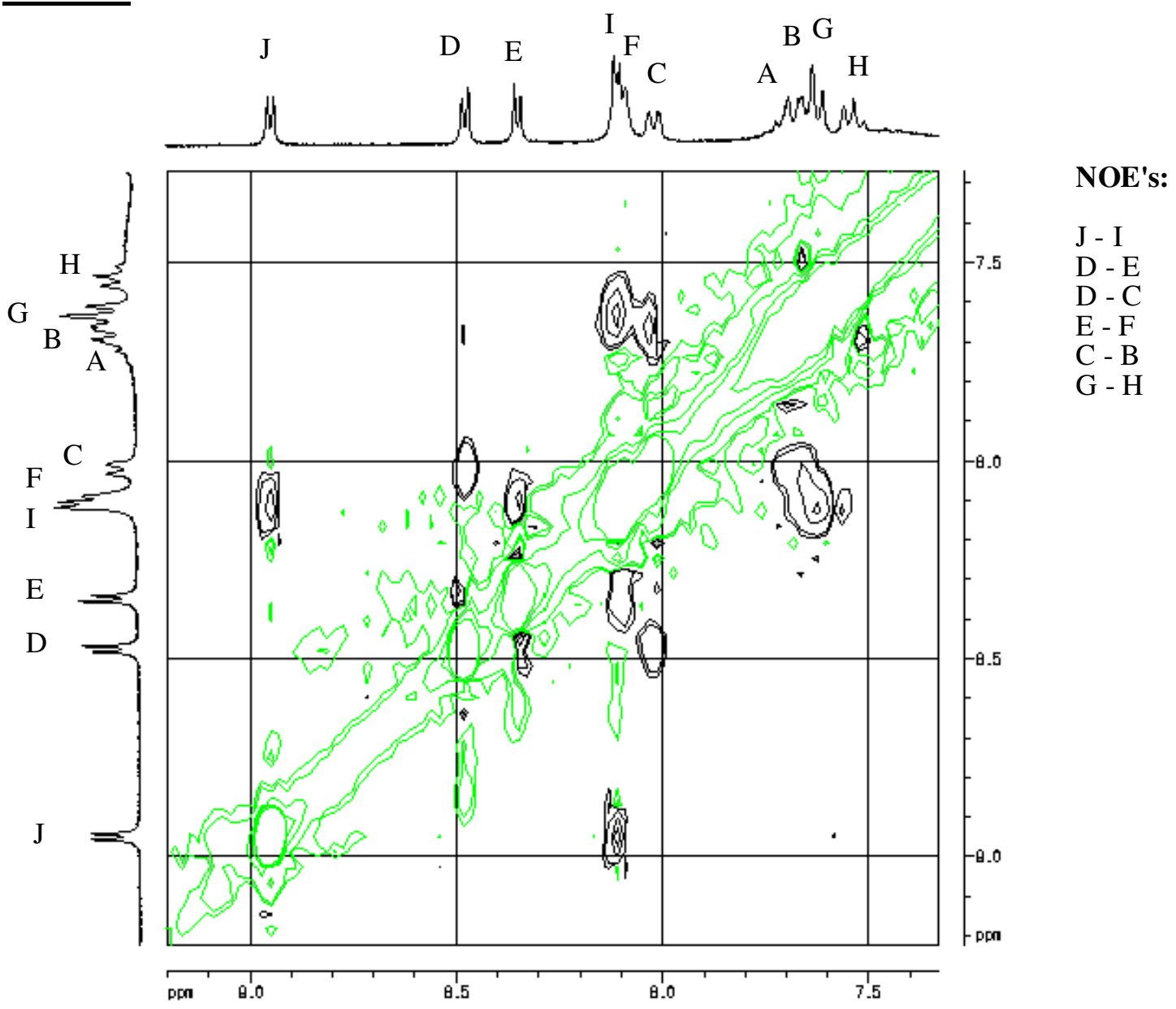




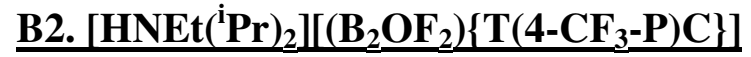

\section{Labeling scheme for assignments:}

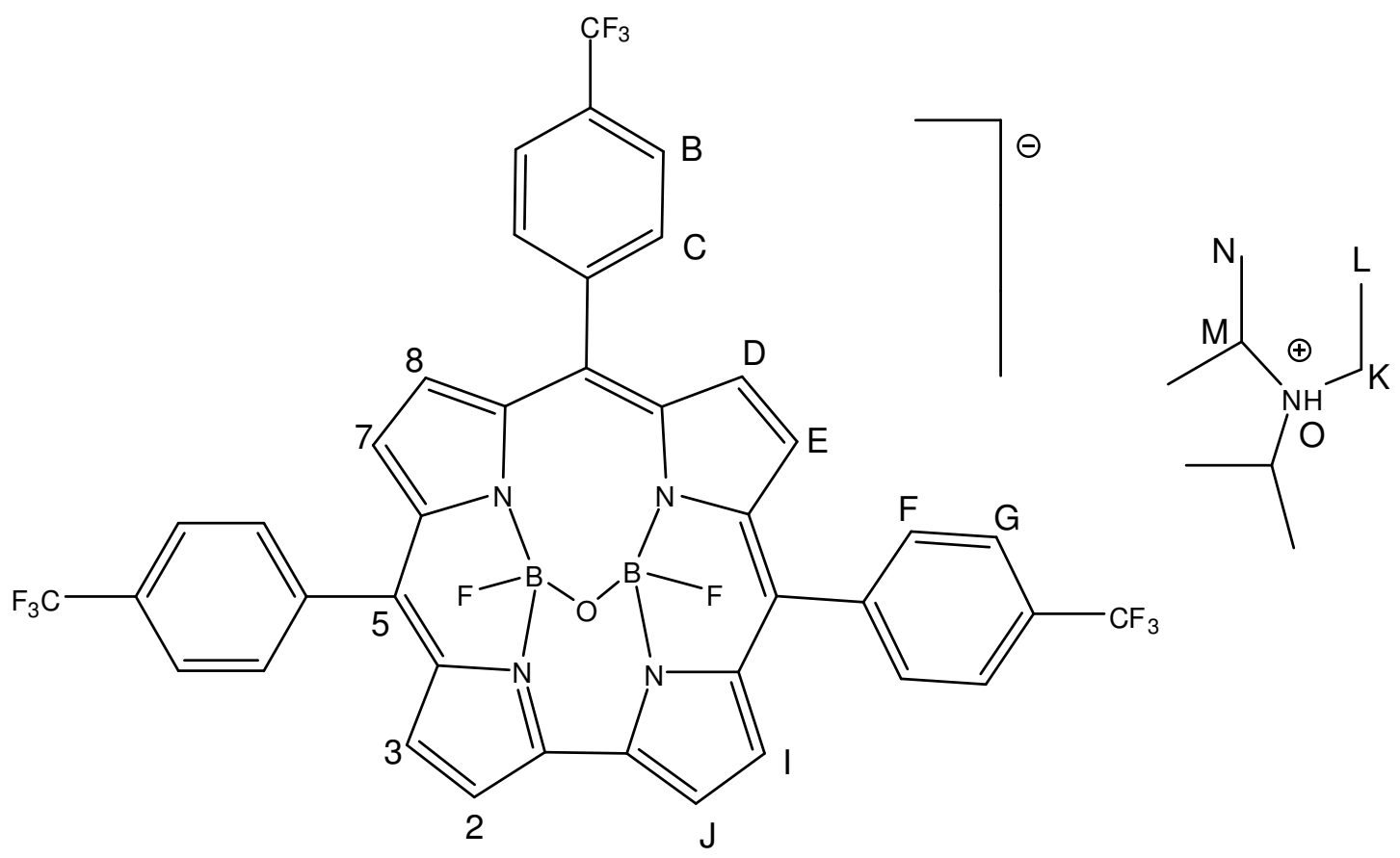

1H NMR:

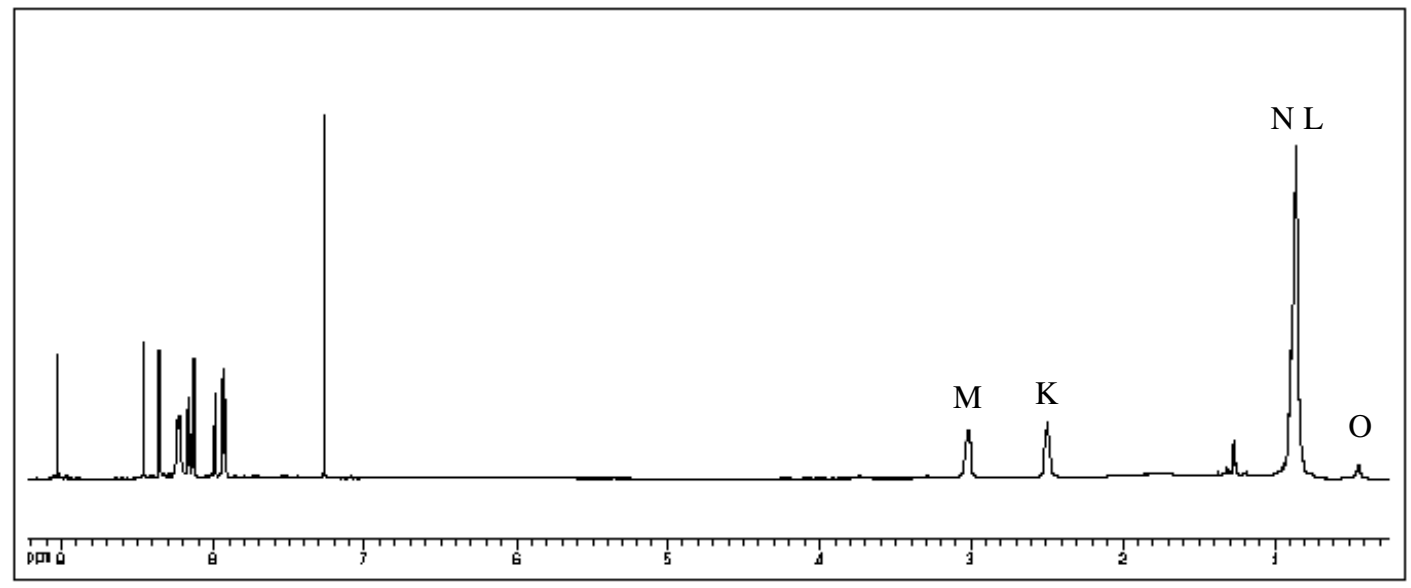


.. $\left[\mathrm{HNEt}\left({ }^{\mathrm{i}} \mathrm{Pr}\right)_{2}\right]\left[\left(\mathrm{B}_{2} \mathrm{OF}_{2}\right)\left\{\mathrm{T}\left(4-\mathrm{CF}_{3}-\mathrm{P}\right) \mathrm{C}\right\}\right]$ continued

${ }^{1}$ H NMR (aromatic region):

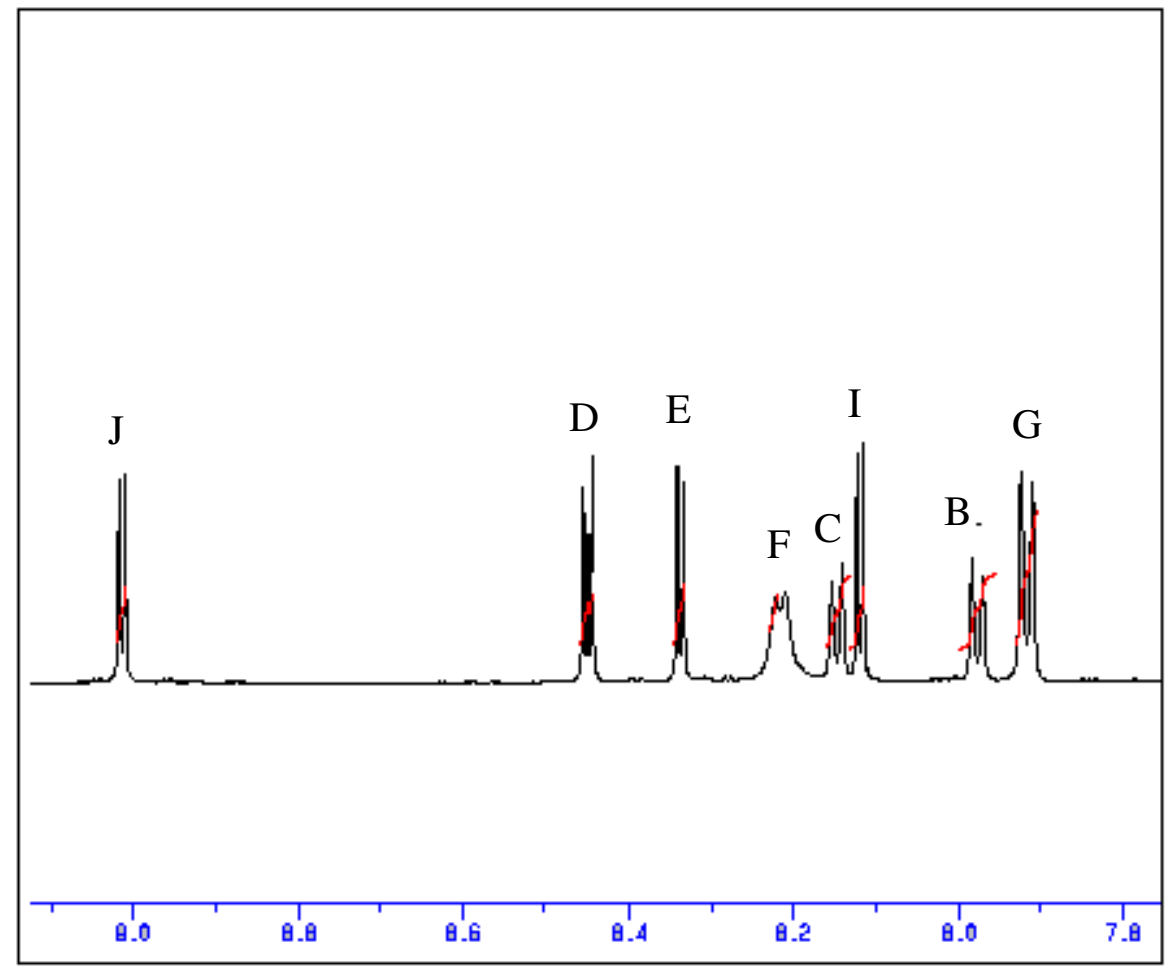


.. $\left[\mathrm{HNEt}\left({ }^{\mathrm{i}} \mathrm{Pr}\right)_{2}\right]\left[\left(\mathrm{B}_{2} \mathrm{OF}_{2}\right)\left\{\mathrm{T}\left(4-\mathrm{CF}_{3}-\mathrm{P}\right) \mathrm{C}\right\}\right]$ continued

\section{COSY:}

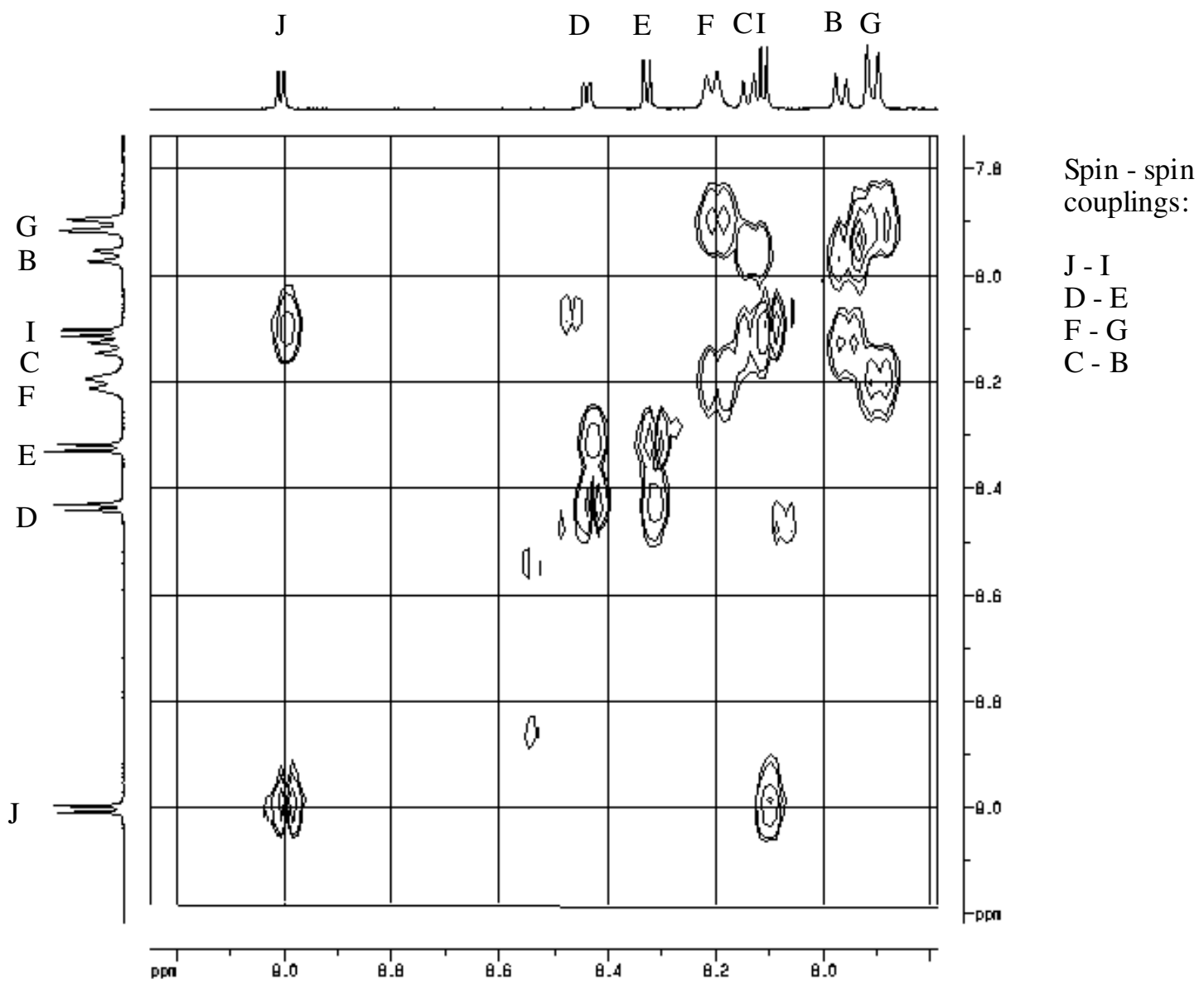




\section{.. $\left[\mathrm{HNEt}\left({ }^{\mathrm{i}} \mathrm{Pr}\right)_{2}\right]\left[\left(\mathrm{B}_{2} \mathrm{OF}{ }_{2}\right)\left\{\mathrm{T}\left(4-\mathrm{CF}_{3}-\mathrm{P}\right) \mathrm{C}\right\}\right]$ continued}

\section{NOESY:}

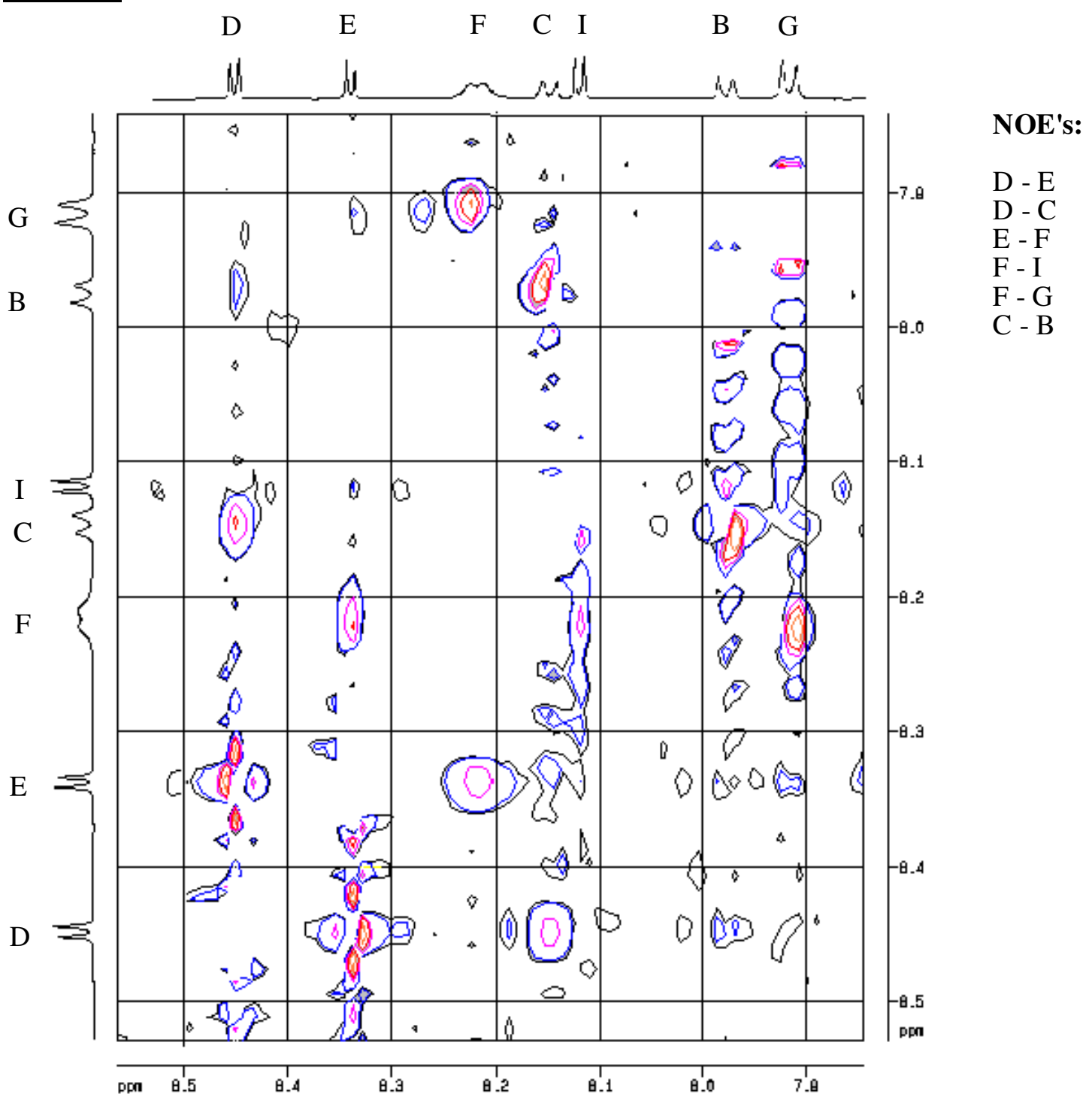


$\left.\left.\underline{\text { B3. }\left[\mathrm{HNEt}\left({ }^{\mathrm{i}} \mathrm{Pr}\right)_{2}\right]\left[\left(\mathrm{B}_{2}\right.\right.} \underline{\mathrm{OF}}_{2}\right)\{\mathrm{T}(4-\mathrm{F}-\mathrm{P}) \mathrm{C}\}\right]$

\section{Labeling scheme for assignments}

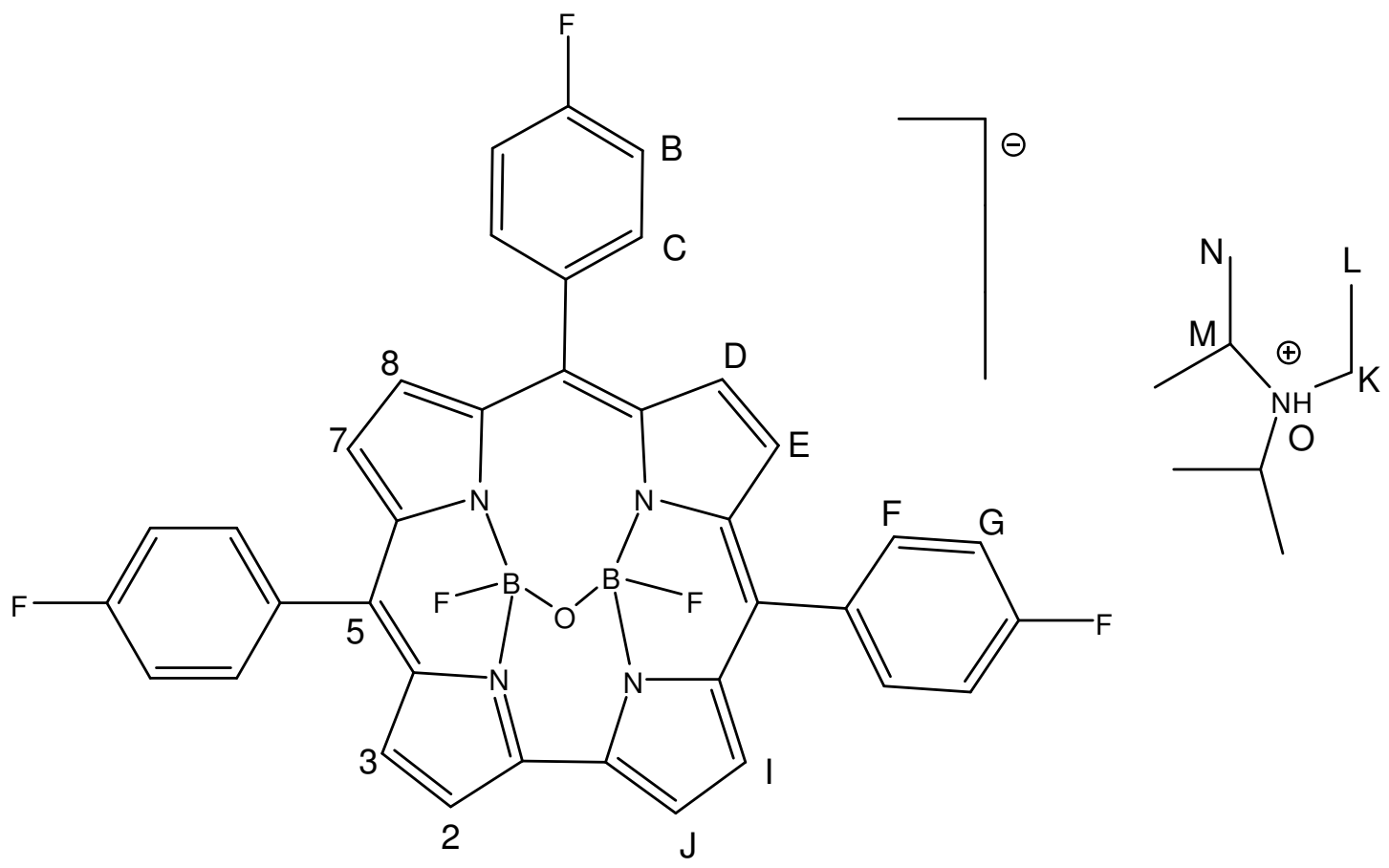

11H NMR:

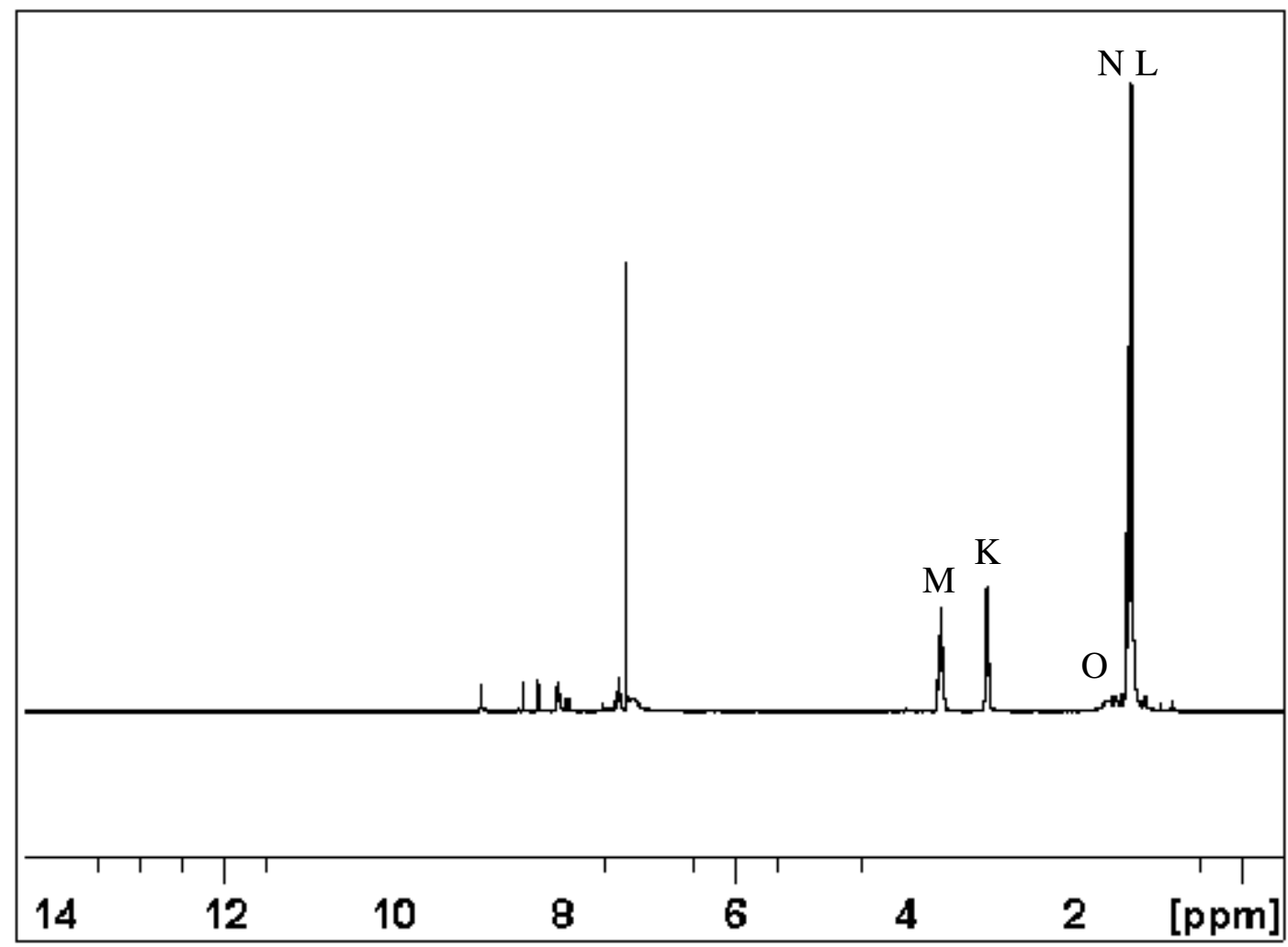


.. $\left[\mathrm{HNEt}\left({ }^{\mathrm{i}} \mathrm{Pr}\right)_{2}\right]\left[\left(\mathrm{B}_{2} \mathrm{OF}{ }_{2}\right)\{\mathrm{T}(4-\mathrm{F}-\mathrm{P}) \mathrm{C}\}\right]$ continued

${ }^{1}$ H NMR (aromatic region):

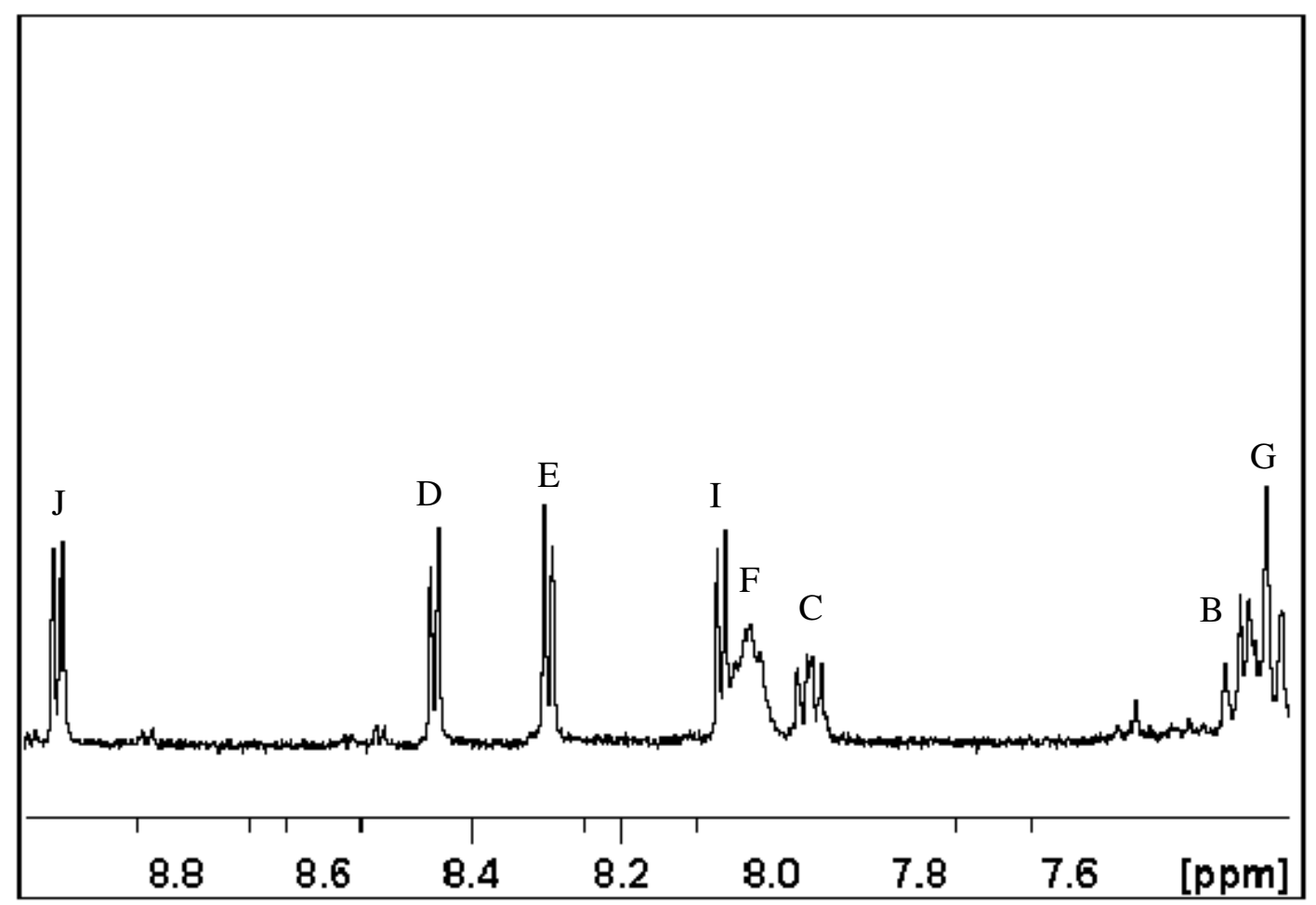


.. $\left[\mathrm{HNEt}\left({ }^{\mathrm{i}} \mathrm{Pr}\right)_{2}\right]\left[\left(\mathrm{B}_{2} \mathrm{OF}{ }_{2}\right)\{\mathrm{T}(4-\mathrm{F}-\mathrm{P}) \mathrm{C}\}\right]$ continued

\section{COSY:}

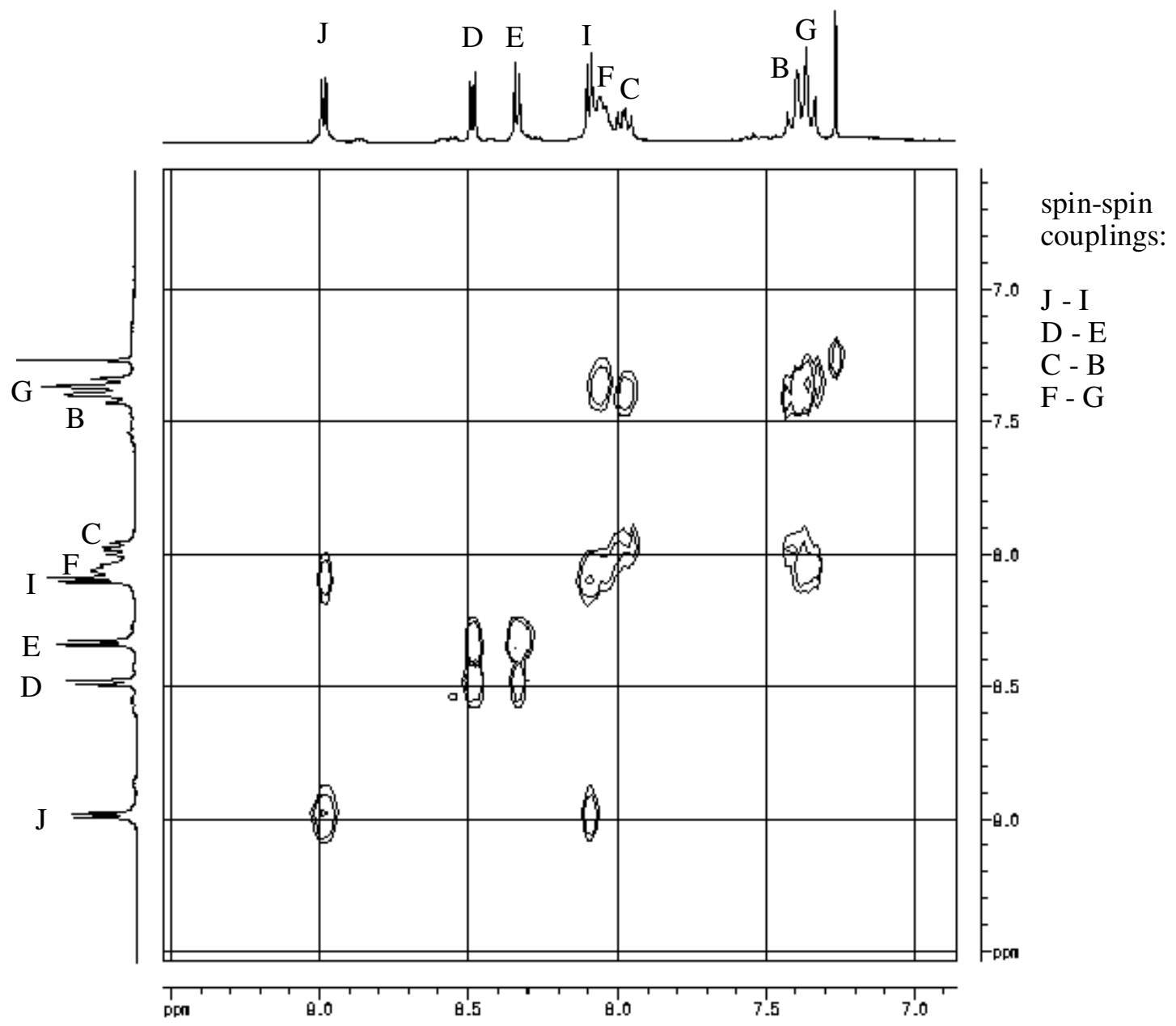


.. $\left[\mathrm{HNEt}\left({ }^{\mathrm{i}} \mathrm{Pr}\right)_{2}\right]\left[\left(\mathrm{B}_{2} \mathrm{OF}{ }_{2}\right)\{\mathrm{T}(4-\mathrm{F}-\mathrm{P}) \mathrm{C}\}\right]$ continued

\section{NOESY:}

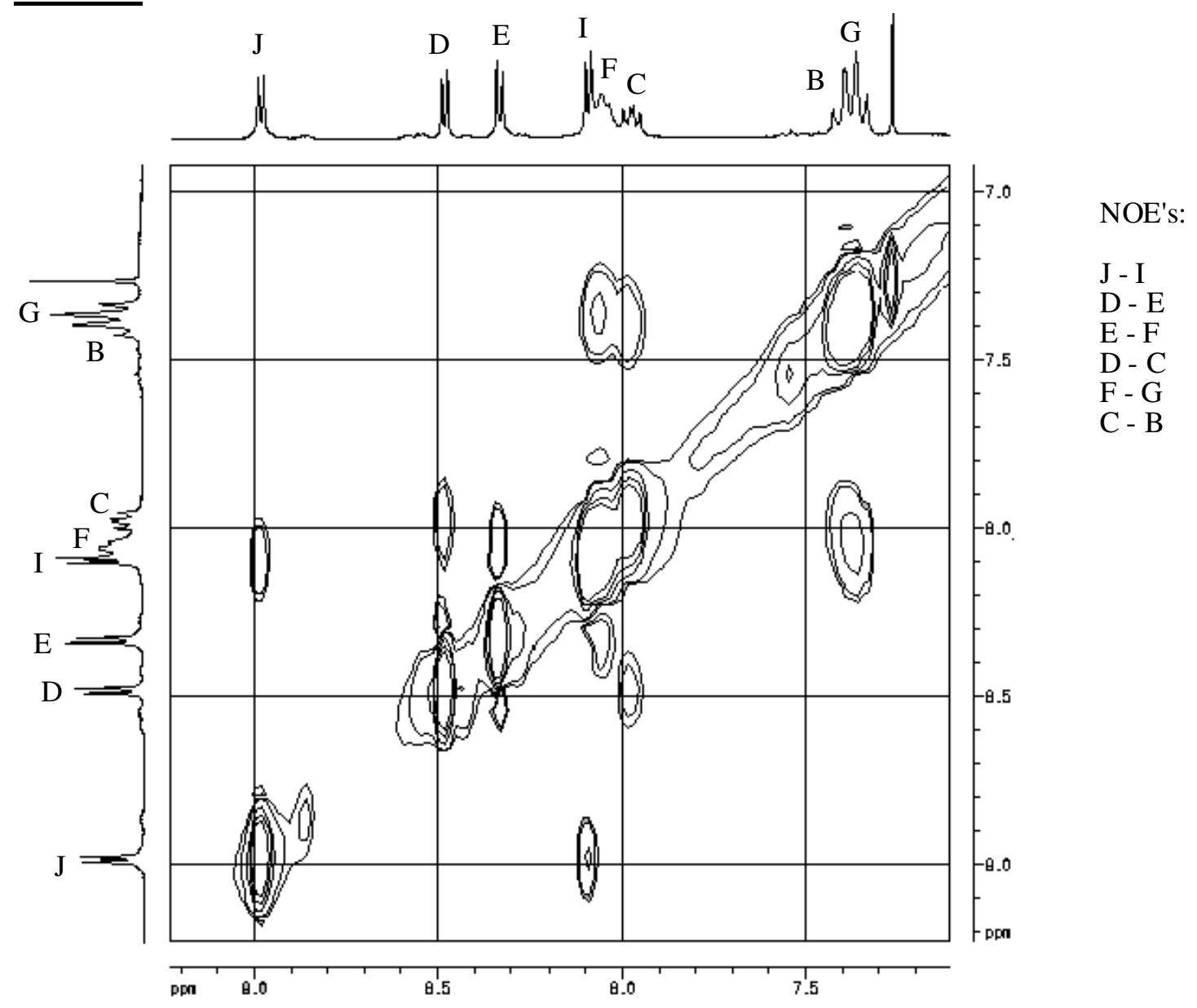




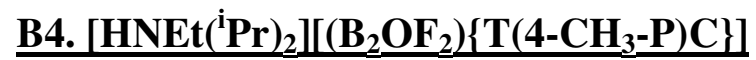

\section{Assignments:}

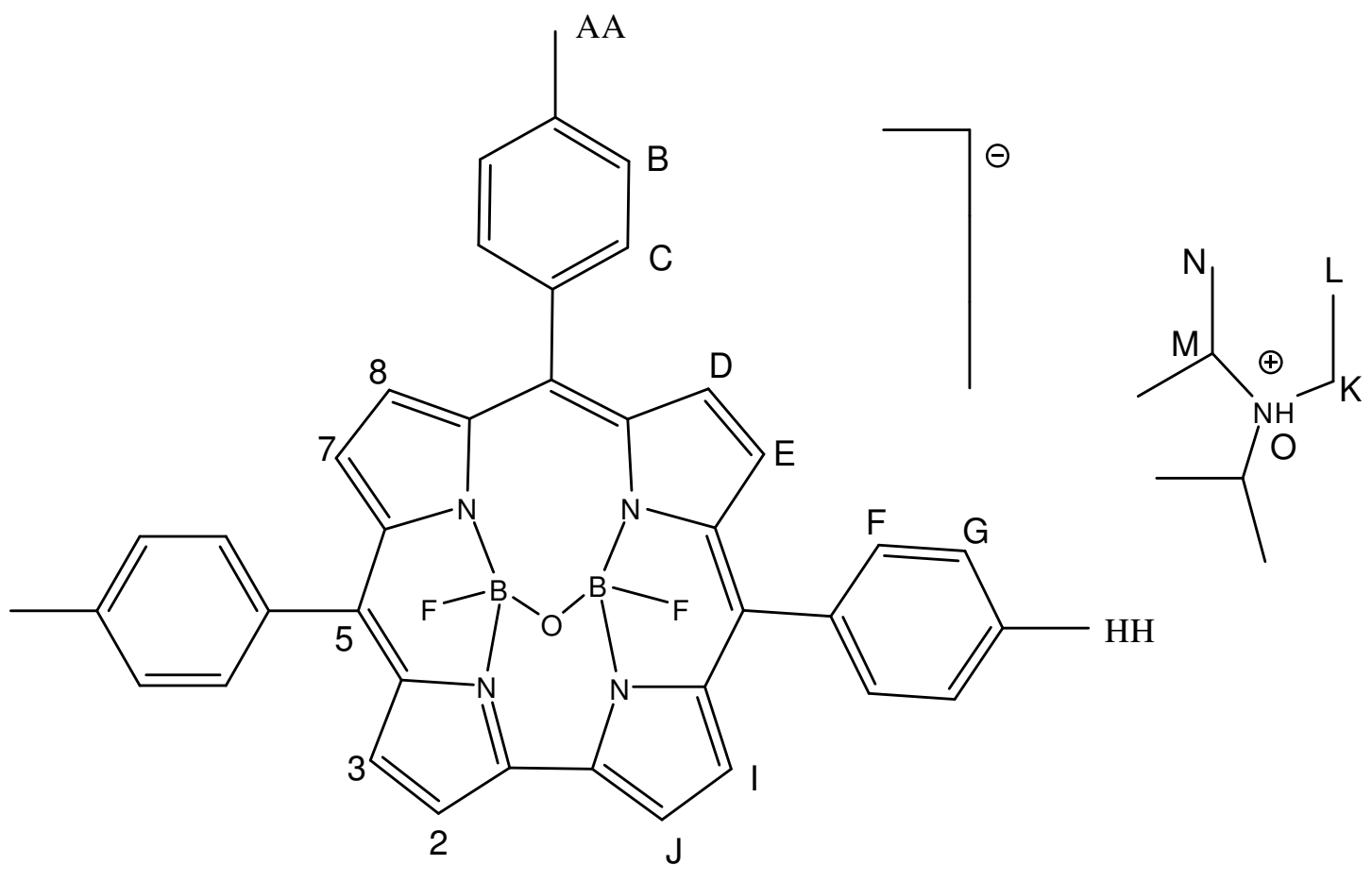

11H NMR:

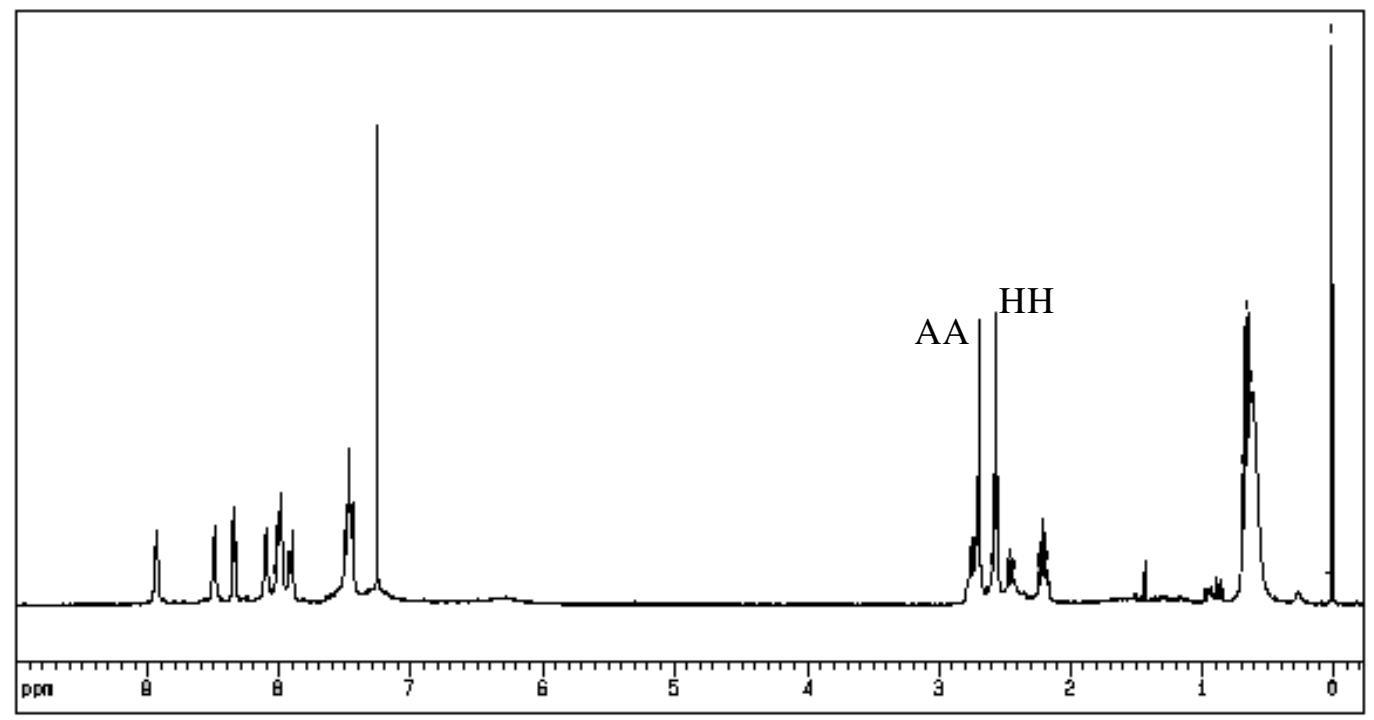


.. $\left[\mathrm{HNEt}\left({ }^{\mathrm{i}} \mathrm{Pr}\right)_{2}\right]\left[\left(\mathrm{B}_{2} \mathrm{OF} 2\right)\left\{\mathrm{T}\left(4-\mathrm{CH}_{3}-\mathrm{P}\right) \mathrm{C}\right\}\right]$ continued

${ }^{1}$ H NMR (aromatic region):

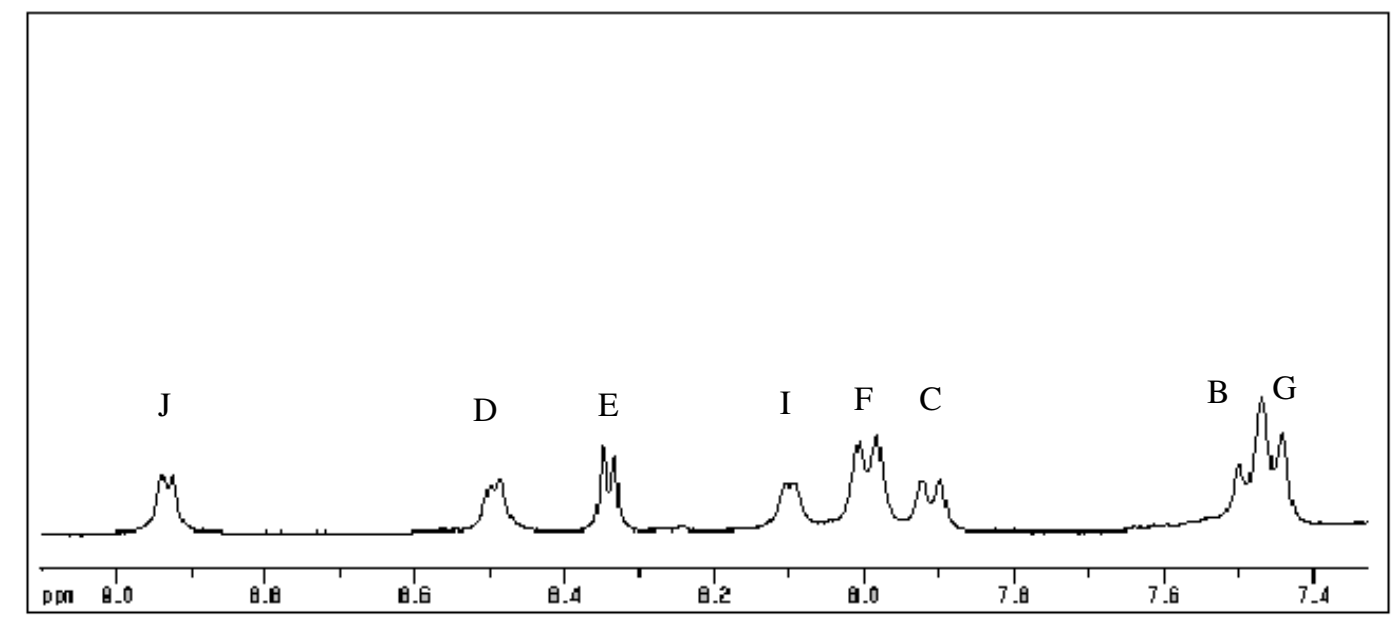

\section{COSY:}

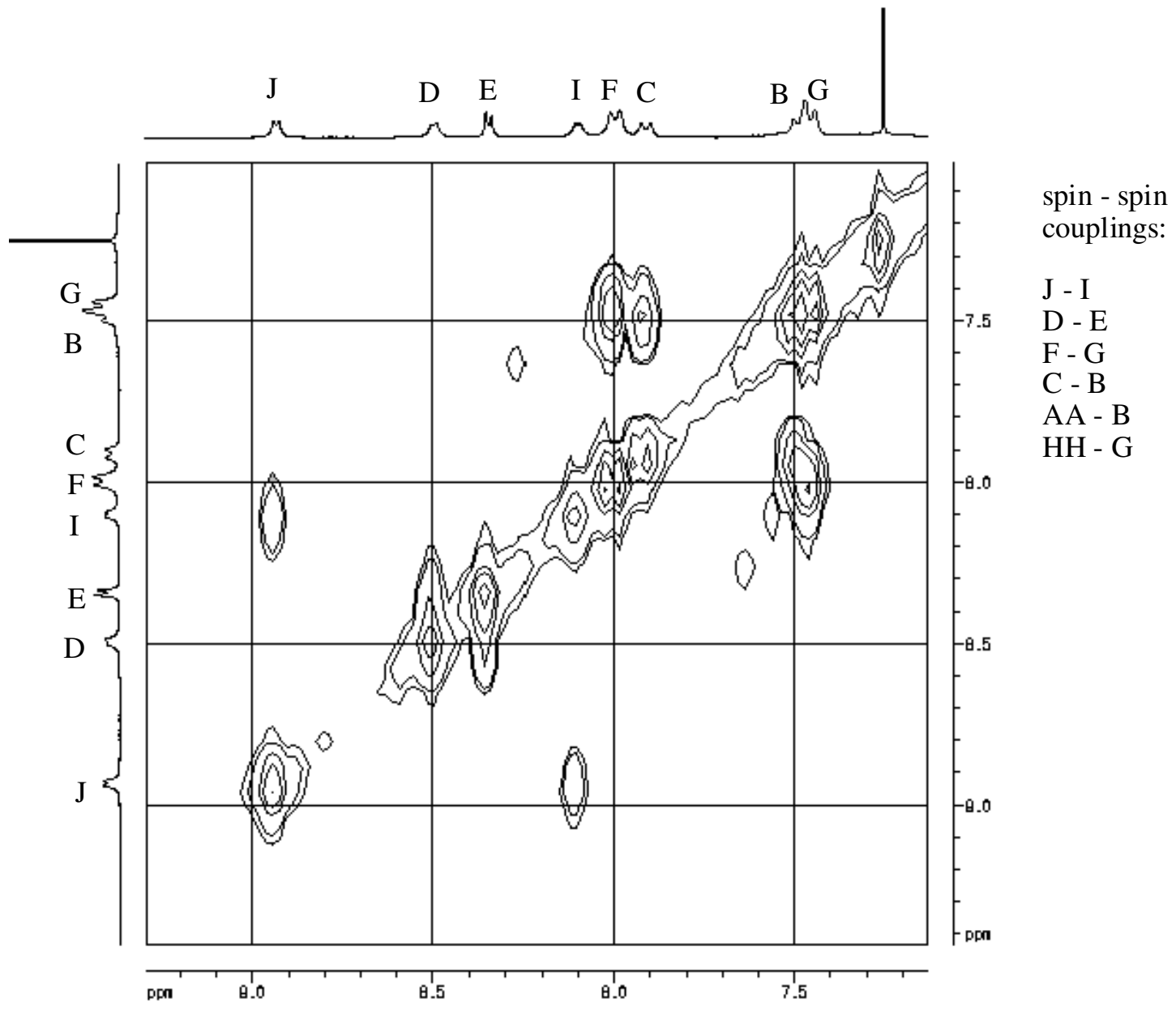


.. $\left[\mathrm{HNEt}\left({ }^{\mathrm{i}} \mathrm{Pr}\right)_{2}\right]\left[\left(\mathrm{B}_{2} \mathrm{OF}_{2}\right)\left\{\mathrm{T}\left(4-\mathrm{CH}_{3}-\mathrm{P}\right) \mathrm{C}\right\}\right]$ continued

\section{NOESY:}

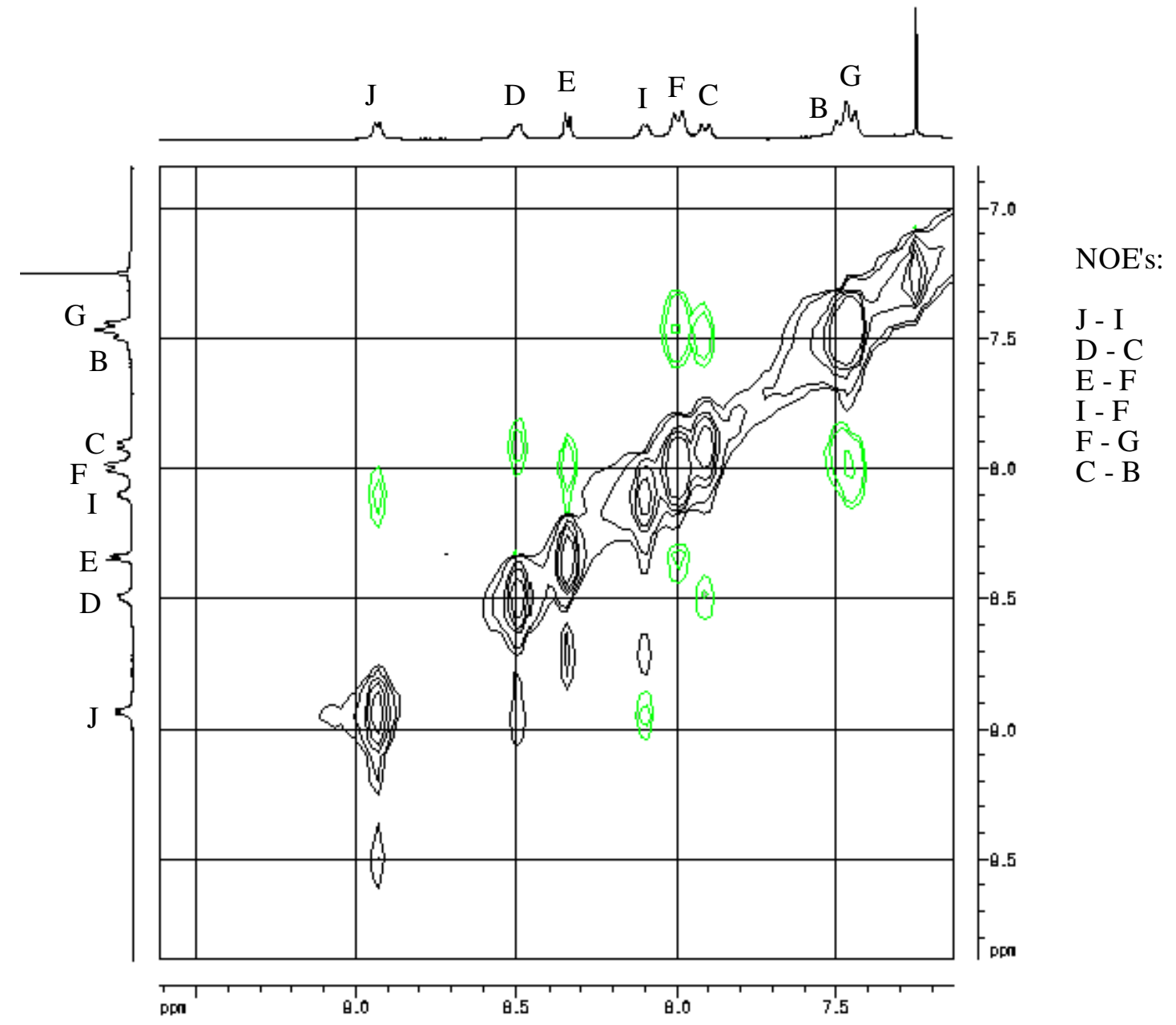


C. UV-vis spectra of the four FBOBF complexes in dichloromethane

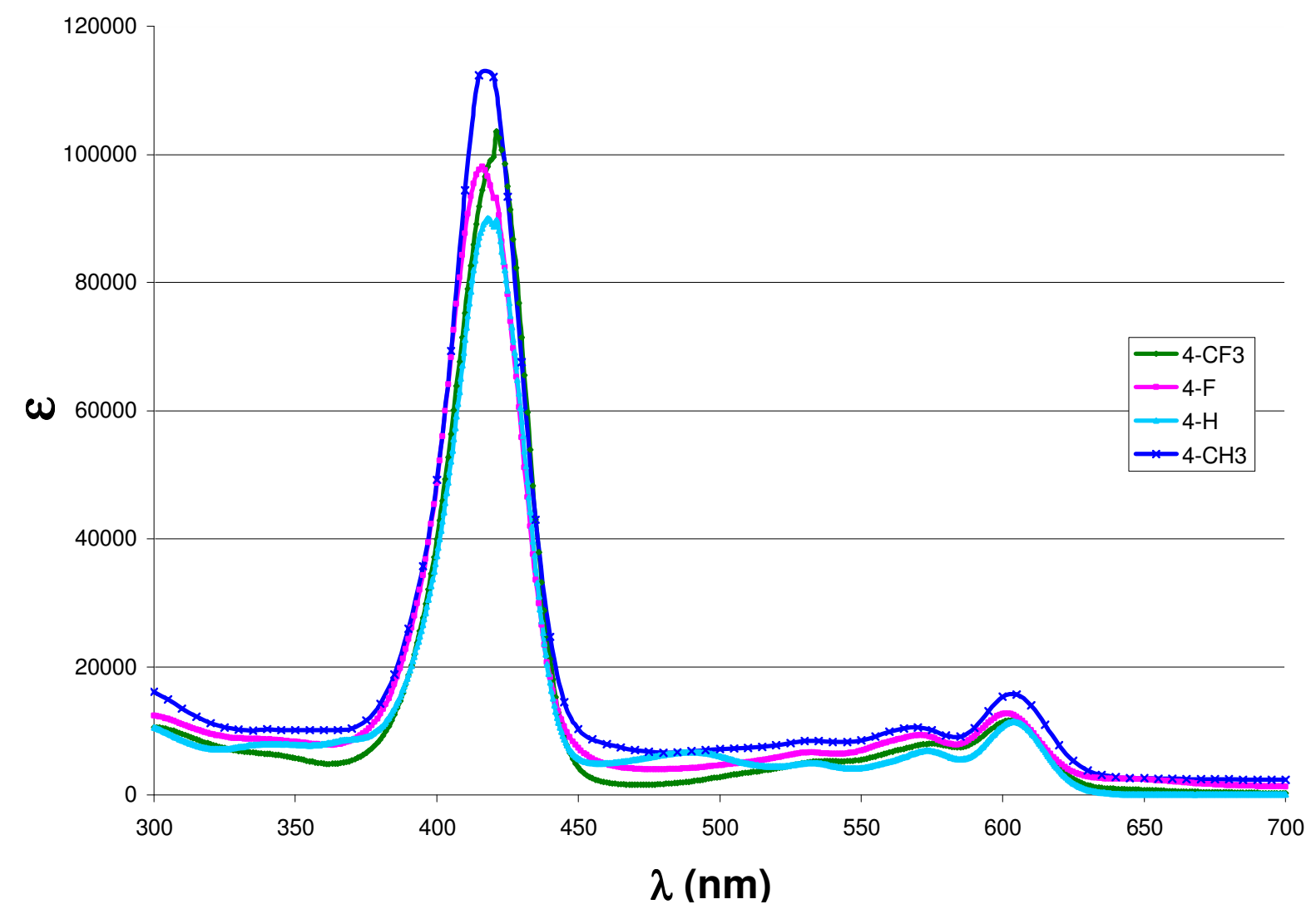




\section{Details of Mass Spectra}

MS Electrospray Ionisation - negative ion mode, solvent: Acetonitrile

\section{$\left[\mathrm{HNEt}\left(\mathrm{Pr}_{2}\right)\right]\left[\left(\mathrm{B}_{2} \underline{\mathrm{OF}}_{2}\right)\left[\mathbf{T}\left(4-\mathrm{CH}_{3}-\mathrm{P}\right) \mathrm{C}\right\}\right]$}

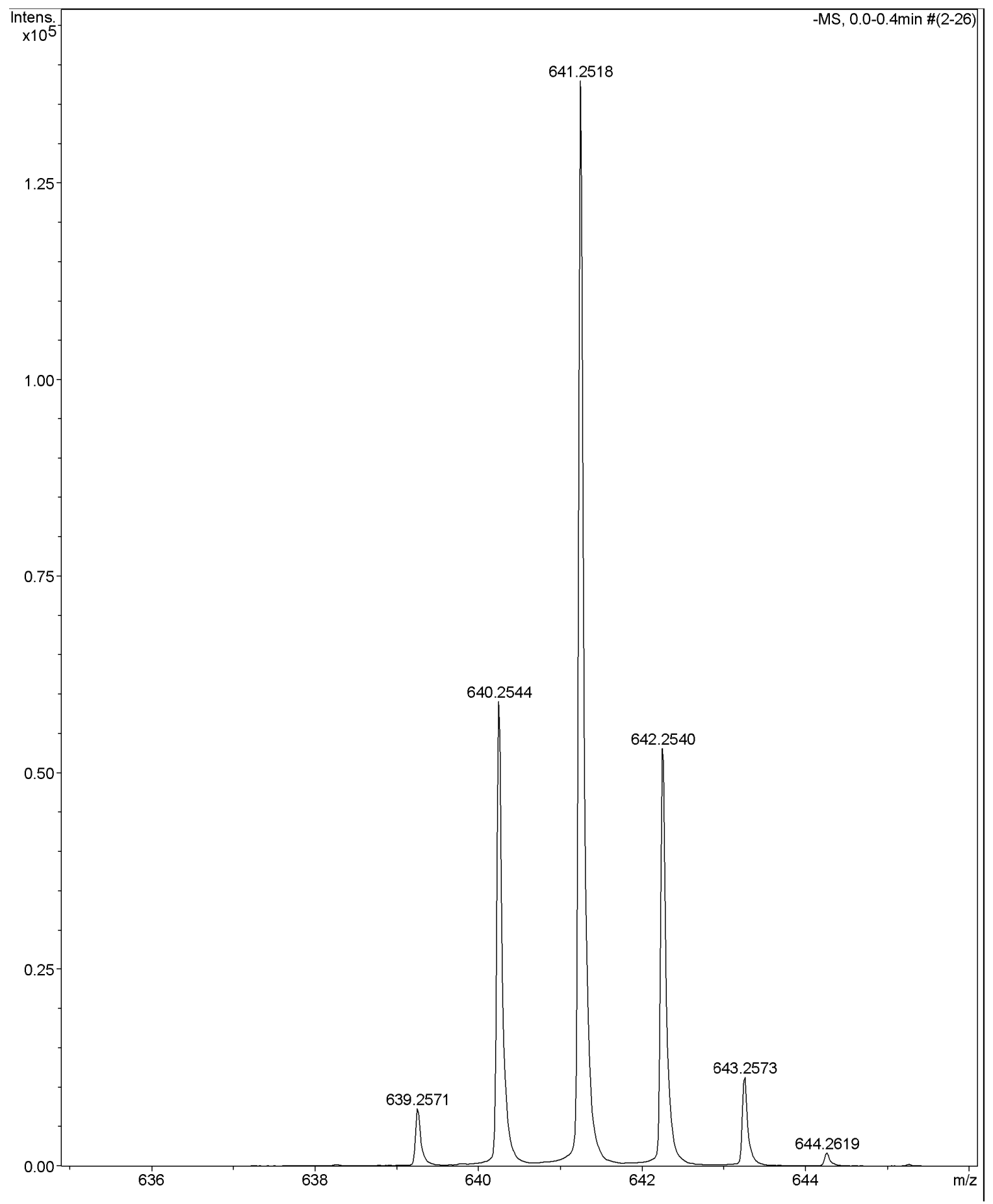


$\left.\left[\operatorname{HNEt}^{\mathrm{i}}{ }^{\mathbf{P r}}{ }_{2}\right)\right]\left[\left(\mathrm{B}_{2} \underline{\mathrm{OF}}_{2}\right)(\mathrm{TPC})\right]$

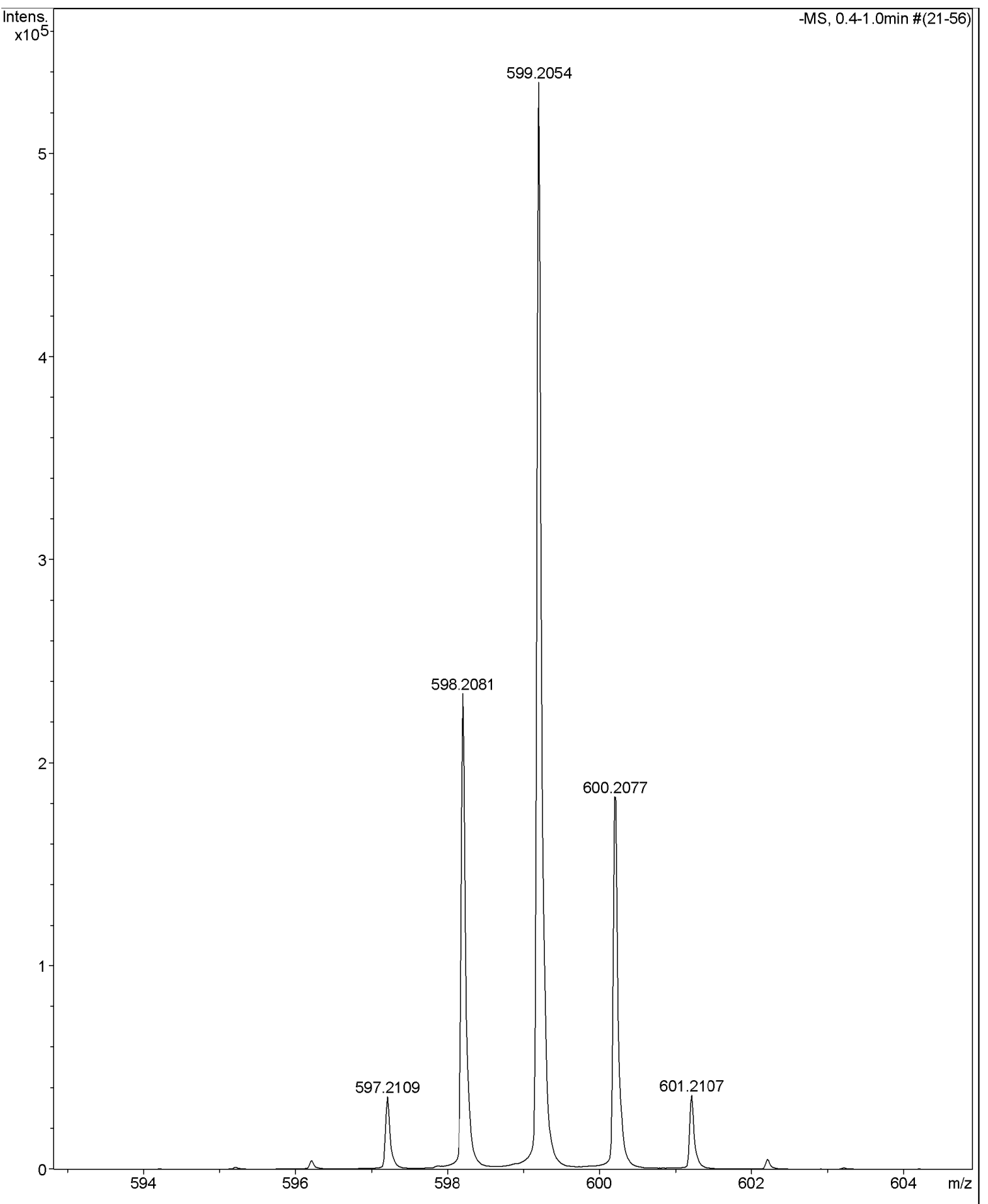




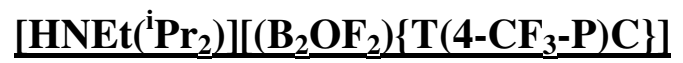

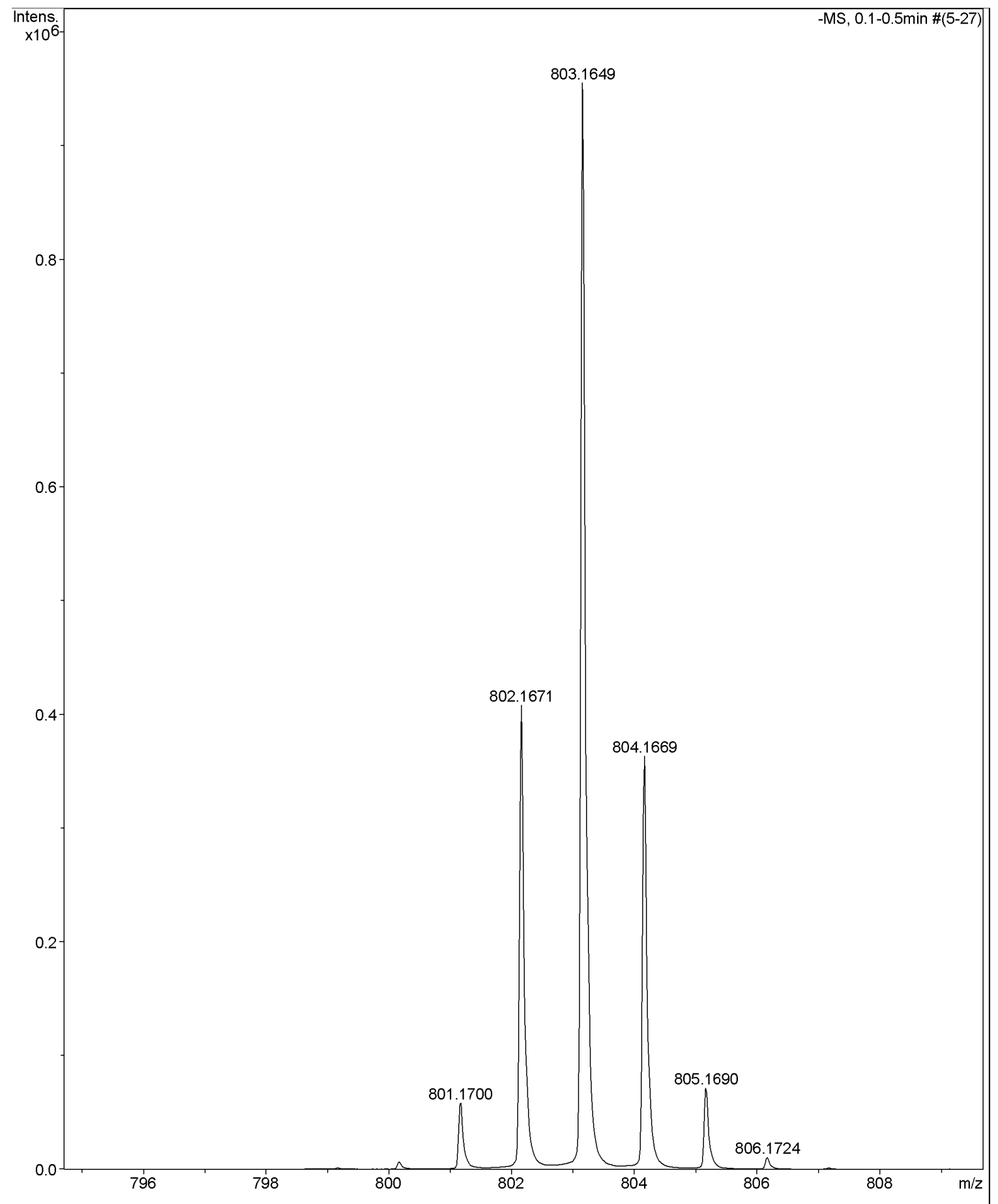




\section{$\left.\left[\operatorname{HNEt}^{\mathrm{i}}{ }^{\mathrm{Pr}_{2}}\right)_{2}\right]\left[\left(\mathrm{B}_{2} \underline{\mathrm{OF}}_{2}\right)\{\mathrm{T}(4-\mathrm{F}-\mathrm{P}) \mathrm{C}\}\right]$}

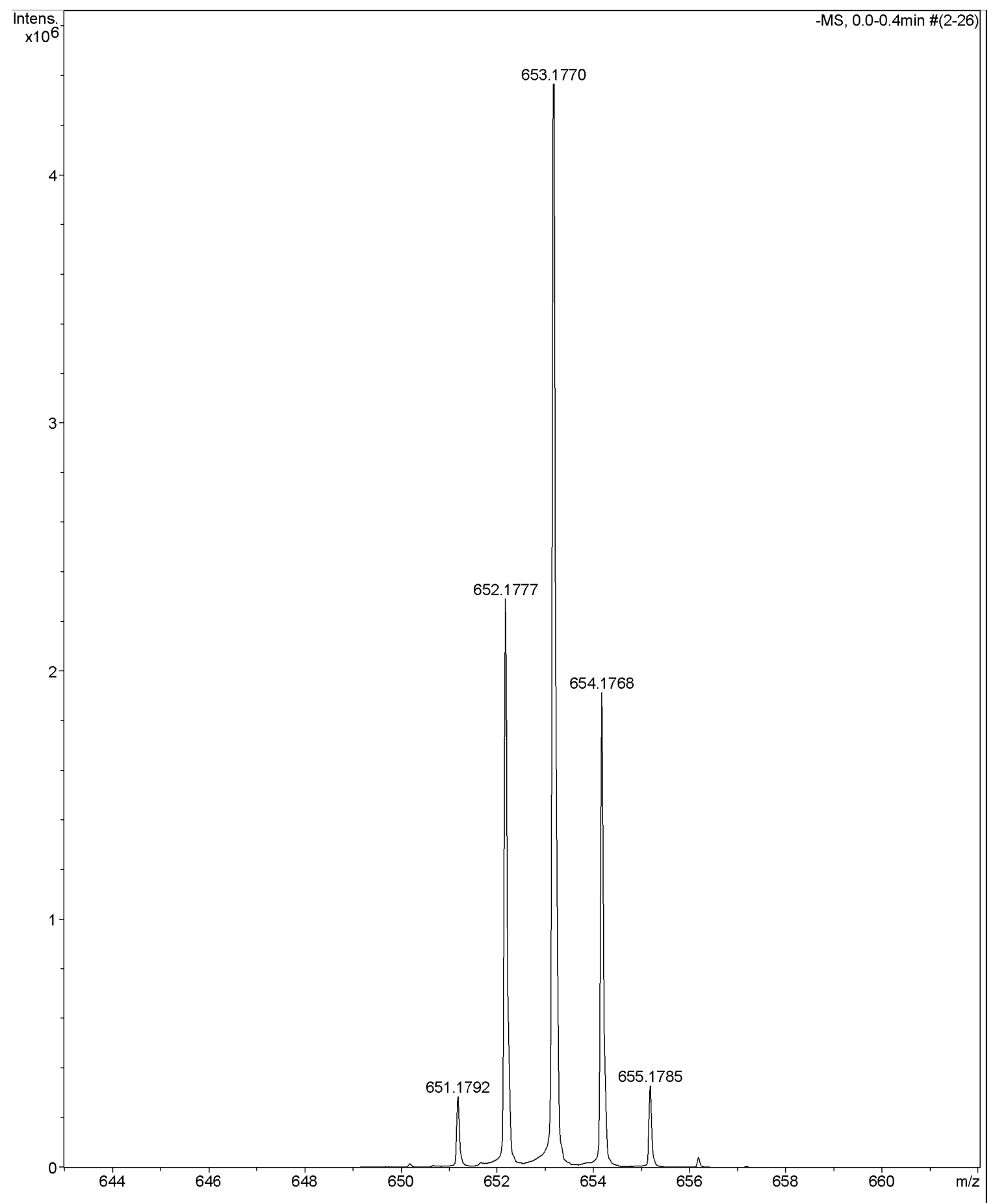




\section{E. Optimized Cartesian coordinates (̊̊)}

All structures were optimized with OLYP/TZ2P. The structures are depicted graphically in Figure 2 of the paper.

1. $\left[\mathrm{B}_{2} \mathrm{OF}_{2}(\text { Corr })\right]^{-}$, cisoid $\mathrm{FBOBF}$ unit coordinated to dipyrromethene binding sites

$\begin{array}{lrrr}\mathrm{H} & -4.134468000 & 1.241375000 & -2.379829000 \\ \mathrm{H} & 4.394747000 & 0.810937000 & 1.263761000 \\ \mathrm{H} & -2.370493000 & 1.558755000 & -4.379804000 \\ \mathrm{H} & 3.273638000 & 1.236572000 & 3.660968000 \\ \mathrm{H} & -4.134468000 & 1.241375000 & 2.379829000 \\ \mathrm{H} & 4.394747000 & 0.810937000 & -1.263761000 \\ \mathrm{H} & 3.273638000 & 1.236572000 & -3.660968000 \\ \mathrm{H} & -2.370493000 & 1.558755000 & 4.379804000 \\ \mathrm{H} & -4.034249000 & 0.516493000 & 0.000000000 \\ \mathrm{H} & 0.477093000 & 1.077956000 & -4.489918000 \\ \mathrm{H} & 0.477093000 & 1.077956000 & 4.489918000 \\ \mathrm{~N} & -1.149262000 & -0.000653000 & -1.720457000 \\ \mathrm{~N} & 1.268891000 & -0.221181000 & 1.469547000 \\ \mathrm{~N} & -1.149262000 & -0.000653000 & 1.720457000 \\ \mathrm{~N} & 1.268891000 & -0.221181000 & -1.469547000 \\ \mathrm{C} & -3.110945000 & 0.884049000 & -2.413032000 \\ \mathrm{C} & 3.395756000 & 0.529144000 & 1.569680000 \\ \mathrm{C} & -2.207960000 & 1.056856000 & -3.432565000 \\ \mathrm{C} & 2.819387000 & 0.751664000 & 2.804777000 \\ \mathrm{C} & -2.429311000 & 0.269181000 & -1.297964000 \\ \mathrm{C} & 2.394604000 & -0.034487000 & 0.712754000 \\ \mathrm{C} & -0.956028000 & 0.531930000 & -2.978425000 \\ \mathrm{C} & 1.456087000 & 0.337735000 & 2.713267000 \\ \mathrm{C} & -2.978584000 & 0.247741000 & 0.000000000 \\ \mathrm{C} & 0.335836000 & 0.625454000 & -3.513129000 \\ \mathrm{C} & 0.335836000 & 0.625454000 & 3.513129000 \\ \mathrm{C} & -2.429311000 & 0.269181000 & 1.297964000 \\ \mathrm{C} & 2.394604000 & -0.034487000 & -0.712754000 \\ \mathrm{C} & 1.456087000 & 0.337735000 & -2.713267000 \\ \mathrm{C} & -0.956028000 & 0.531930000 & 2.978425000 \\ \mathrm{C} & -3.110945000 & 0.884049000 & 2.413032000 \\ \mathrm{C} & 3.395756000 & 0.529144000 & -1.569680000 \\ \mathrm{C} & 2.819387000 & 0.751664000 & -2.804777000 \\ \mathrm{C} & -2.207960000 & 1.056856000 & 3.432565000 \\ \mathrm{~B} & -0.065489000 & -1.057744000 & -1.256666000 \\ \mathrm{~B} & -0.065489000 & -1.057744000 & 1.256666000 \\ \mathrm{O} & -0.253328000 & -1.602917000 & 0.000000000 \\ \mathrm{~F} & -0.055072000 & -2.082612000 & -2.236185000 \\ \mathrm{~F} & -0.055072000 & -2.082612000 & 2.236185000\end{array}$


2. $\left[\mathrm{B}_{2} \mathrm{OF}_{2}(\mathrm{Cor} r)\right]^{-}$, cisoid $\mathrm{FBOBF}$ unit coordinated to dipyrromethene/dipyrrole binding sites

$\begin{array}{lrrr}\mathrm{H} & 4.047877000 & -1.538796000 & -2.814611000 \\ \mathrm{H} & -4.373162000 & -1.648328000 & 1.830753000 \\ \mathrm{H} & 2.015262000 & -0.847880000 & -4.433433000 \\ \mathrm{H} & -2.765105000 & -1.172885000 & 3.930144000 \\ \mathrm{H} & 4.047877000 & -1.538796000 & 2.814611000 \\ \mathrm{H} & -4.373162000 & -1.648328000 & -1.830753000 \\ \mathrm{H} & -2.765105000 & -1.172885000 & -3.930144000 \\ \mathrm{H} & 2.015262000 & -0.847880000 & 4.433433000 \\ \mathrm{H} & 4.362689000 & -1.392743000 & 0.000000000 \\ \mathrm{H} & -0.286129000 & -0.171926000 & -4.161081000 \\ \mathrm{H} & -0.286129000 & -0.171926000 & 4.161081000 \\ \mathrm{~N} & 1.524156000 & -0.030143000 & -1.258117000 \\ \mathrm{~N} & -1.743191000 & 0.226000000 & 1.144450000 \\ \mathrm{~N} & 1.524156000 & -0.030143000 & 1.258117000 \\ \mathrm{~N} & -1.743191000 & 0.226000000 & -1.144450000 \\ \mathrm{C} & 3.136578000 & -1.017782000 & -2.546414000 \\ \mathrm{C} & -3.478838000 & -1.037751000 & 1.836515000 \\ \mathrm{C} & 2.104925000 & -0.652701000 & -3.371426000 \\ \mathrm{C} & -2.654569000 & -0.772502000 & 2.929046000 \\ \mathrm{C} & 2.754843000 & -0.650005000 & -1.218734000 \\ \mathrm{C} & -2.867871000 & -0.425490000 & 0.717135000 \\ \mathrm{C} & 1.049312000 & -0.088632000 & -2.563680000 \\ \mathrm{C} & -1.514904000 & -0.036114000 & 2.455420000 \\ \mathrm{C} & 3.394139000 & -0.904362000 & 0.000000000 \\ \mathrm{C} & -0.249916000 & 0.015502000 & -3.089933000 \\ \mathrm{C} & -0.249916000 & 0.015502000 & 3.089933000 \\ \mathrm{C} & 2.754843000 & -0.650005000 & 1.218734000 \\ \mathrm{C} & -2.867871000 & -0.425490000 & -0.717135000 \\ \mathrm{C} & -1.514904000 & -0.036114000 & -2.455420000 \\ \mathrm{C} & 1.049312000 & -0.088632000 & 2.563680000 \\ \mathrm{C} & 3.136578000 & -1.017782000 & 2.546414000 \\ \mathrm{C} & -3.478838000 & -1.037751000 & -1.836515000 \\ \mathrm{C} & -2.654569000 & -0.772502000 & -2.929046000 \\ \mathrm{C} & 2.104925000 & -0.652701000 & 3.371426000 \\ \mathrm{~B} & -1.246681000 & 1.211842000 & 0.000000000 \\ \mathrm{~B} & 1.256332000 & 0.946227000 & 0.000000000 \\ \mathrm{O} & 0.061279000 & 1.635960000 & 0.000000000 \\ \mathrm{~F} & 2.331045000 & 1.868438000 & 0.000000000 \\ \mathrm{~F} & -2.122825000 & 2.324556000 & 0.000000000 \\ & & & \end{array}$


3. $\left[\mathrm{B}_{2} \mathrm{OF}_{2}(\mathrm{Corr})\right]^{-}$, transoid $\mathrm{FBOBF}$ unit coordinated to dipyrromethene binding sites

$\begin{array}{lrrr}\mathrm{H} & -4.286317000 & 0.796402000 & -2.589073000 \\ \mathrm{H} & 4.393788000 & 1.002702000 & 1.299102000 \\ \mathrm{H} & -2.563152000 & 0.861637000 & -4.627837000 \\ \mathrm{H} & 3.299977000 & 1.367828000 & 3.715679000 \\ \mathrm{H} & -4.042425000 & 1.517923000 & 2.388802000 \\ \mathrm{H} & 4.530704000 & 0.283265000 & -1.450158000 \\ \mathrm{H} & 3.389496000 & 0.520122000 & -3.855320000 \\ \mathrm{H} & -2.335608000 & 1.741943000 & 4.435967000 \\ \mathrm{H} & -4.004153000 & 0.680049000 & 0.077652000 \\ \mathrm{H} & 0.465019000 & 0.654772000 & -4.630391000 \\ \mathrm{H} & 0.520173000 & 1.136072000 & 4.576292000 \\ \mathrm{~N} & -1.182196000 & 0.276542000 & -1.718010000 \\ \mathrm{~N} & 1.316645000 & -0.147151000 & 1.552934000 \\ \mathrm{~N} & -1.158825000 & 0.025967000 & 1.868661000 \\ \mathrm{~N} & 1.265240000 & 0.115967000 & -1.396750000 \\ \mathrm{C} & -3.214265000 & 0.649867000 & -2.528707000 \\ \mathrm{C} & 3.412758000 & 0.674214000 & 1.618397000 \\ \mathrm{C} & -2.322802000 & 0.686519000 & -3.585938000 \\ \mathrm{C} & 2.846304000 & 0.868939000 & 2.867518000 \\ \mathrm{C} & -2.475137000 & 0.400548000 & -1.307815000 \\ \mathrm{C} & 2.422528000 & 0.094901000 & 0.762161000 \\ \mathrm{C} & -1.000925000 & 0.465311000 & -3.051076000 \\ \mathrm{C} & 1.494427000 & 0.408498000 & 2.792954000 \\ \mathrm{C} & -2.945988000 & 0.423910000 & 0.038653000 \\ \mathrm{C} & 0.315130000 & 0.473836000 & -3.571311000 \\ \mathrm{C} & 0.365197000 & 0.669257000 & 3.608187000 \\ \mathrm{C} & -2.384433000 & 0.410989000 & 1.358362000 \\ \mathrm{C} & 2.426925000 & 0.097252000 & -0.686239000 \\ \mathrm{C} & 1.452436000 & 0.314408000 & -2.730330000 \\ \mathrm{C} & -0.947093000 & 0.579895000 & 3.102210000 \\ \mathrm{C} & -3.051684000 & 1.084200000 & 2.455495000 \\ \mathrm{C} & 3.467854000 & 0.265146000 & -1.658031000 \\ \mathrm{C} & 2.874981000 & 0.387149000 & -2.911010000 \\ \mathrm{C} & -2.175057000 & 1.203700000 & 3.509214000 \\ \mathrm{~B} & -0.084332000 & 0.145010000 & -0.714061000 \\ \mathrm{~B} & -0.051175000 & -0.930322000 & 1.294701000 \\ \mathrm{O} & -0.275990000 & -1.105335000 & -0.051370000 \\ \mathrm{~F} & -0.107153000 & 1.379565000 & 0.006542000 \\ \mathrm{~F} & -0.023559000 & -2.111498000 & 2.059941000 \\ & & & \end{array}$


4. $\left[\mathrm{B}_{2} \mathrm{OF}_{2} \text { (Corr) }\right]^{-}$, transoid $\mathrm{FBOBF}$ unit coordinated to dipyrromethene/dipyrrole binding sites

$\begin{array}{lrrr}\mathrm{H} & 4.237075000 & 0.649378000 & 2.825576000 \\ \mathrm{H} & -4.816694000 & 1.196482000 & -2.033267000 \\ \mathrm{H} & 2.036137000 & 0.609496000 & 4.349259000 \\ \mathrm{H} & -3.105972000 & 0.828506000 & -4.051406000 \\ \mathrm{H} & 4.237075000 & 0.649378000 & -2.825576000 \\ \mathrm{H} & -4.816694000 & 1.196482000 & 2.033267000 \\ \mathrm{H} & -3.105972000 & 0.828506000 & 4.051406000 \\ \mathrm{H} & 2.036137000 & 0.609496000 & -4.349259000 \\ \mathrm{H} & 4.586966000 & 0.230048000 & 0.000000000 \\ \mathrm{H} & -0.305353000 & 0.257334000 & 4.088270000 \\ \mathrm{H} & -0.305353000 & 0.257334000 & -4.088270000 \\ \mathrm{~N} & 1.537994000 & -0.482349000 & 1.272958000 \\ \mathrm{~N} & -1.854839000 & 0.178082000 & -1.127394000 \\ \mathrm{~N} & 1.537994000 & -0.482349000 & -1.272958000 \\ \mathrm{~N} & -1.854839000 & 0.178082000 & 1.127394000 \\ \mathrm{C} & 3.250733000 & 0.300335000 & 2.543895000 \\ \mathrm{C} & -3.789485000 & 0.866220000 & -1.941429000 \\ \mathrm{C} & 2.122327000 & 0.273703000 & 3.322847000 \\ \mathrm{C} & -2.889061000 & 0.670531000 & -3.001094000 \\ \mathrm{C} & 2.858034000 & -0.129127000 & 1.231478000 \\ \mathrm{C} & -3.094474000 & 0.556908000 & -0.735191000 \\ \mathrm{C} & 0.992846000 & -0.140464000 & 2.514373000 \\ \mathrm{C} & -1.626875000 & 0.242409000 & -2.452535000 \\ \mathrm{C} & 3.540818000 & -0.058688000 & 0.000000000 \\ \mathrm{C} & -0.325443000 & 0.055870000 & 3.018587000 \\ \mathrm{C} & -0.325443000 & 0.055870000 & -3.018587000 \\ \mathrm{C} & 2.858034000 & -0.129127000 & -1.231478000 \\ \mathrm{C} & -3.094474000 & 0.556908000 & 0.735191000 \\ \mathrm{C} & -1.626875000 & 0.242409000 & 2.452535000 \\ \mathrm{C} & 0.992846000 & -0.140464000 & -2.514373000 \\ \mathrm{C} & 3.250733000 & 0.300335000 & -2.543895000 \\ \mathrm{C} & -3.789485000 & 0.866220000 & 1.941429000 \\ \mathrm{C} & -2.889061000 & 0.670531000 & 3.001094000 \\ \mathrm{C} & 2.122327000 & 0.273703000 & -3.322847000 \\ \mathrm{~B} & -0.880896000 & -0.012509000 & 0.000000000 \\ \mathrm{~B} & 1.001201000 & -1.291477000 & 0.000000000 \\ \mathrm{O} & -0.372433000 & -1.332239000 & 0.000000000 \\ \mathrm{~F} & 1.625342000 & -2.551498000 & 0.000000000 \\ \mathrm{~F} & -0.026930000 & 1.120204000 & 0.000000000 \\ & & & \end{array}$

